\title{
IDENTIFICAÇÃO DE ALGUNS CONSTITUINTES QUÍMICOS DE Indigofera hirsuta LINN. (FABACEAE) POR CLAE-IES-EM (TOF) E AVALIAÇÃO DA ATIVIDADE ANTIRRADICALAR
}

\author{
Adriana Candido da Silva Moura*, Wagner Vilegas e Lourdes Campaner dos Santos \\ Departamento de Química Orgânica, Instituto de Química, Universidade Estadual Paulista, CP 355, 14800-900 Araraquara - SP, Brasil
}

Recebido em 14/7/10; aceito em 21/2/11; publicado na web em 15/4/11

\begin{abstract}
IDENTIFICATION OF SOME CHEMICAL CONSTITUENTS OF Indigofera hirsuta Linn. (FABACEAE) BY HPLC-ESI-MS (TOF) AND EVALUATION OF THE ANTIRADICAL ACTIVITY. A rapid analytical approach, suitable to characterize the compounds present in the aqueous and methanol extracts prepared from the aerial parts of Indigofera hirsute, was developed. The method based on high-performance liquid chromatography coupled to mass spectrometry, electrospray positive ionization and detection by time of flight (HPLC-ESI-MS-TOF) identified, tryptophan, uracil, rutin, kaempferol-3-O- $\beta$-D-glucopyranoside, gallic acid and methyl gallate. The antiradical activity of this extract was evaluated using DPPH assay, with gallic acid as antiradical pattern. The study revealed the antiradical activity of methyl galatte $\left(\mathrm{EC}_{50}=5 \pm 0.3 \mu \mathrm{g} \mathrm{mL}-1\right)$ galic acid $\left(\mathrm{EC}_{50}=5 \pm 0.2 \mu \mathrm{g} \mathrm{mL}^{-1}\right)$ and rutin $\left(\mathrm{EC}_{50}=21.6 \pm 0.6 \mu \mathrm{g} \mathrm{m}\right.$ $\left.\mathrm{L}^{-1}\right)$, isolated from methanol extract $\left(\mathrm{EC}_{50}=67.7 \pm 0.9 \mu \mathrm{g} \mathrm{mL}-1\right)$, which showed strong antiradical activity.
\end{abstract}

Keywords: Indigofera hirsuta; mass spectrometry; antiradical activity.

\section{INTRODUÇÃO}

Muitos dos benefícios proporcionados pelas plantas medicinais são atribuídos a substâncias com propriedade antirradicalar, principalmente substâncias fenólicas encontrados em inúmeras plantas. ${ }^{1}$ Essas substâncias protegem o organismo de reações oxidativas que podem desencadear uma série de doenças no organismo humano. ${ }^{2}$

A família Fabaceae, também conhecida como Leguminosae, possui ampla distribuição nas regiões temperadas e tropicais. É subdividida em 3 subfamílias muito distintas: Faboideae, Caesalpinioideae e Mimosoideae. ${ }^{3}$

Para a subfamília Faboideae estão descritos aproximadamente 482 gêneros e cerca de 12000 espécies de ampla distribuição nas regiões temperadas e tropicais, sendo que algumas dessas espécies tem grande importância medicinal, como as pertencentes ao gênero Indigofera. ${ }^{4}$

O gênero Indigofera apresenta cerca de 700 espécies herbáceas e arbustivas que ocorrem em regiões tropicais e subtropicais, principalmente na África, ocorrendo na Austrália, Argentina, China, Índia, Panamá, sudeste asiático e Tailândia. No Brasil foram identificadas 11 espécies, sendo algumas delas amplamente distribuídas no estado de São Paulo. ${ }^{5,6}$

Espécies de Indigofera apresentam uma diversidade de classes de substâncias, sendo encontrados flavonoides derivados do kaempferol em I. hebepetala ${ }^{7,8}$ e rotenoides em I. tictoria. ${ }^{9}$ Ácidos fenólicos e flavonoides foram encontrados em I. mysorensis ${ }^{10} \mathrm{e}$ em I. heterantha. ${ }^{11-13}$ De I. microcarpa foram isolados dois arilbenzofuranos. ${ }^{14}$ Nitrocompostos alifáticos ocorrem em mais de 60 espécies de Indigofera. ${ }^{15}$ São também encontrados glicosídeos do ácido 3-nitropropanoico (NPA), ${ }^{16}$ bem como seus ésteres mono-, di-, tri- e tetrassubstituídos. ${ }^{17-19} \mathrm{De}$ Indigofera hirsuta foi isolado o kaempferol 3,5-digalactosídeo, ${ }^{20} \mathrm{com}$ atividade antiparasitária. ${ }^{21}$

O presente trabalho foi realizado com o objetivo de determinar a composição química do extrato aquoso e do extrato metanólico

\footnotetext{
*e-mail: adrianacsmoura@yahoo.com.br
}

das partes aéreas de Indigofera hirsuta por CLAE-IES-EM (TOF), bem como avaliar a atividade antirradicalar do precipitado (obtido da centrifugação do extrato aquoso), dos extratos aquoso e metanólico e das substâncias isoladas.

\section{PARTE EXPERIMENTAL}

\section{Procedimentos cromatográficos}

Para os procedimentos cromatográficos foram utilizados: placas preparadas de sílica gel $60\left(\operatorname{Merck}^{\circledR}\right)(20$ x $20 \mathrm{~cm}$ x 0,2 mm); coluna de vidro preenchida com Sephadex LH-20 (70 cm x 3,0 cm d.i.), acoplada a uma bomba peristáltica modelo P1 18-1110-91 (Pharmacia ${ }^{\circledR}$ ) e a um coletor automático Redifrac (Pharmacia ${ }^{\circledR}$ ); cromatógrafo líquido de média pressão Büchi Pump Manager C-615; cromatógrafo líquido de alta eficiência (analítico) modelo PU-2089 ( Jasco $^{\circledR}$ ), acoplado a detector de arranjo de foto diodos com faixa de varredura de $195-650 \mathrm{~nm}$ e intervalo mínimo de $1 \mathrm{~nm}$, modelo MD-20140 (Jasco $\left.{ }^{\circledR}\right)$, com coluna de fase reversa $\mathrm{RP}_{18}$ imobilizadas com octadecilsilano, modelo Luna 2 $\left(\right.$ Phenomenex $\left.{ }^{\circledR}\right)(250 \times 4,6 \mathrm{~mm}$ d.i.) com partículas de tamanho médio de $5 \mu \mathrm{m}$ e softwares Star Chromatography Workstation versão 5.31 (Varian ${ }^{\circledR}$ ) e EZChrom Elite Client/Server versão 3.1.7 (Chromatec ${ }^{\circledR}$ ) para o processamento dos dados cromatográficos; cromatógrafo líquido de alta eficiência (semipreparativo) modelo ProStar 210/330 $\left(\right.$ Varian $^{\circledR}$ ) acoplado a detector de arranjo de foto diodos, com coluna de fase reversa $\mathrm{RP}_{18}$ imobilizadas com octadecilsilano, modelo Luna $2\left(\right.$ Phenomenex $\left.^{\circledR}\right)(250 \times 10,0 \mathrm{~mm}$ d.i. $)$ com partículas de tamanho médio de $10 \mu \mathrm{m}$.

\section{Determinação estrutural}

Para obtenção dos dados espectroscópicos e espectrométricos foram utilizados: espectrômetro de ressonância magnética nuclear de $11,7 \mathrm{~T}$ (Varian Inova), operando a $500 \mathrm{MHz}$ para ${ }^{1} \mathrm{H}$ e $125 \mathrm{MHz}$ para ${ }^{13} \mathrm{C}$, TMS foi usado como padrão interno para determinação dos deslocamentos químicos; espectrofotômetro de infravermelho 
Jasco $^{\circledast}$, modelo FT/IR - 4100; espectrofotômetro de ultravioleta/ visível $\left(\right.$ Hach $\left.^{\circledR}\right)$, modelo DR/4000 U; espectrômetro de massas de alta resolução (EM-IES-QTOF) (FCF-USP-RP) ultrOTOFQ-ESI-TOF Bruker Daltonics ${ }^{\circledR}$ (Billerica, MA - USA) acoplado ao cromatógrafo líquido de alta eficiência Shimadzu ${ }^{\circledR}$ equipado com detector de arranjo de foto diodos e coluna de fase reversa $C_{18}$ Shimadzu $^{\circledR}(250$ x 4,6 mm d.i.) com partículas de tamanho médio de $5 \mu \mathrm{m}$.

\section{Material vegetal}

As partes aéreas de Indigofera hirsuta Linn. foram coletadas em dezembro de 2007 no Estado do Tocantins, Brasil, pelo botânico E. R. dos Santos. Uma exsicata, de número 389, foi depositada no herbário da Universidade Federal do Tocantins (UFT).

\section{Extração e isolamento}

Após a coleta, o material vegetal foi seco em estufa a $40{ }^{\circ} \mathrm{C}$ e triturado em moinho de facas. $\mathrm{O}$ extrato aquoso foi preparado utilizando-se $400 \mathrm{~g}$ desse material e $4 \mathrm{~L}$ de água a $80{ }^{\circ} \mathrm{C}$ em erlenmeyer durante $24 \mathrm{~h}$. A solução foi filtrada e armazenada a $10^{\circ} \mathrm{C}$, ocorrendo formação de precipitado, o qual foi separado por centrifugação $(1 \mathrm{~g})$. O sobrenadante foi em seguida concentrado e liofilizado, resultando em $99 \mathrm{~g}$ de extrato aquoso. O extrato metanólico (40 g) foi obtido a partir de $200 \mathrm{~g}$ do material vegetal por percolação com metanol seguido de destilação do solvente sob pressão reduzida.

O extrato aquoso $(3 \mathrm{~g}, 4 \mathrm{x})$ foi solubilizado em metanol e fracionado por cromatografia de permeação em gel (CPG) em coluna de Sephadex LH-20 eluída com MeOH. As frações obtidas foram analisadas por cromatografia em camada delgada comparativa (CCDC) [placas de sílica gel, eluídas em $\mathrm{CHCl}_{3} / \mathrm{MeOH} / n-\mathrm{PrOH} / \mathrm{H}_{2} \mathrm{O}$, (5:6:1:4, fase orgânica), reveladas com solução de anisaldeído/ $\left.\mathrm{H}_{2} \mathrm{SO}_{4}\right]^{22}$ e agrupadas, resultando em 7 frações.

A fração $3(241 \mathrm{mg})$ foi purificada por cromatografia líquida média pressão (CLMP) $\left(\mathrm{C}_{18}, 15\right.$ x 1,5 cm, gradiente: $\mathrm{H}_{2} \mathrm{O} / \mathrm{MeOH}$; 2,5 $\left.\mathrm{mL} \mathrm{min}^{-1}\right)$, resultando nas substâncias: triptofano $(\mathbf{1}, 18 \mathrm{mg})$ e uracila $(2,12 \mathrm{mg})$.

O extrato metanólico (1,5 g) foi fracionado por cromatografia líquida de alta eficiência (CLAE) (coluna Luna 2 (Phenomenex ${ }^{\circledR}$ ) $10 \mu \mathrm{m}(250 \times 10,0 \mathrm{~mm}), 254 \mathrm{~nm}, 4 \mathrm{~mL} \mathrm{m^{-1 }}$, gradiente: solvente A: $\mathrm{H}_{2} \mathrm{O}$ acidificado com $0,05 \%$ de TFA, solvente $\mathrm{B}$ : $\mathrm{MeOH}$ acidificado com $0,05 \%$ de TFA, $10 \%$ B - 40\% B (10 min), 40\% B - 60\% B (30 $\min ), 60 \% \mathrm{~B}-100 \% \mathrm{~B}$ (35 min); $100 \mathrm{mg} \mathrm{mL}^{-1}$; injeções de $80 \mu \mathrm{L}$ ). Este fracionamento resultou no isolamento dos flavonoides rutina (3, $12 \mathrm{mg}$ ) e kaempferol-3-O- $\beta$-D-glucopiranosídeo (4, $9 \mathrm{mg})$, além de ácido gálico $(\mathbf{5}, 15 \mathrm{mg})$ e galato de metila $(\mathbf{6}, 10 \mathrm{mg})$.

O precipitado ( $1 \mathrm{~g}$ ) foi fracionado por CPG, fornecendo 10 frações, que foram analisadas por CCDC (eluídas e reveladas nas mesmas condições que o extrato aquoso), resultando no isolamento do flavonoide kaempferol $(7,100 \mathrm{mg})$. A purificação por CLAE da fração 9 permitiu o isolamento do ácido protocatecuico $(\mathbf{8}, 7 \mathrm{mg})$ [coluna Luna 2 (Phenomenex $^{\circledR}$ ) $10 \mu \mathrm{m}$ (250 x $\left.10 \mathrm{~mm}\right), 254 \mathrm{~nm}, 4,8$ $\mathrm{mL} \mathrm{min}^{-1}$, gradiente: solvente $\mathrm{A}: \mathrm{H}_{2} \mathrm{O}$ acidificado com $0,05 \%$ de TFA, solvente B: ACN acidificado com $0,05 \%$ de TFA, $5 \%$ B - 10\% B (30 $\min ), 10 \% \mathrm{~B}-100 \% \mathrm{~B}(50 \mathrm{~min}) ; 80 \mathrm{mg} \mathrm{mL}^{-1}$; injeções de $\left.80 \mu \mathrm{L}\right]$.

\section{Análise dos extratos por CLAE-DAD-IES-EM (TOF)}

Os extratos metanólico e aquoso foram, separadamente, solubilizados em $\mathrm{MeOH} / \mathrm{H}_{2} \mathrm{O}\left(5: 95\right.$, v/v) $\left(20 \mathrm{mg} \mathrm{mL}^{-1}\right)$, filtrados em membrana de PTFE (politetrafluoretileno) e submetidos à análise por cromatografia líquida de alta eficiência acoplada a detector de arranjos de diodos e espectrômetro de massas - ionização por electrospray e detecção de íons por tempo de vôo [CLAE-DAD-IES-EM (TOF)]. Foi utilizado sistema CLAE-DAD $\left(\right.$ Shimadzu $\left.^{\circledR}\right)\left(\right.$ Coluna Shimadzu $^{\circledR}$ $\mathrm{NST}_{18} 5 \mu \mathrm{m}(250 \times 4,60 \mathrm{~mm}), 254 \mathrm{~nm}, 1 \mathrm{~mL} \mathrm{~min}^{-1}$, gradiente: solvente A: $\mathrm{H}_{2} \mathrm{O}$ acidificado com $0,05 \%$ de TFA, solvente $\mathrm{B}$ : $\mathrm{MeOH}$ acidificado com $0,05 \%$ de TFA, $5 \%$ B - 100\% B (60 min). Os extratos foram analisados no modo positivo de ionização.

\section{Atividade antirradicalar}

A atividade sequestradora de radicais livres dos extratos e das substâncias isoladas foi determinada utilizando DPPH (2,2-difenil1-picrilidrazila). ${ }^{23-25}$

Para avaliar a atividade sequestradora de radicais livres foi utilizada uma solução $0,04 \mathrm{mg} \mathrm{mL}^{-1}$ de DPPH em metanol. Foram adicionados $2 \mathrm{~mL}$ desta solução a $1 \mathrm{~mL}$ da solução de cada amostra em diferentes concentrações $\left(5,10,20,40,80\right.$ e $\left.160 \mu \mathrm{g} \mathrm{mL}^{-1}\right)$. Após 30 min foram medidas as absorbâncias das soluções resultantes em $517 \mathrm{~nm}$. O teste foi realizado em triplicata. O ácido gálico comercial $\left(\right.$ Sigma $\left.^{\circledR}\right)$ foi utilizado como padrão ${ }^{26}\left(\mathrm{CE}_{50}=5 \pm 0,2 \mu \mathrm{g} \mathrm{mL}^{-1}\right)$. As porcentagens de absorbância foram medidas pela equação $\left(\% \Delta_{0}\right)=$ $100 \times\left(\mathrm{A}_{0}-\mathrm{A}\right) / \mathrm{A}_{0}$. A capacidade de doar um hidrogênio radicalar das amostras foi estabelecida por análise de regressão linear no intervalo de confiança de $95 \%(\mathrm{P}<0,05)$ pelo programa Excel 2007, expressando a concentração eficiente $\left(\mathrm{CE}_{50}\right)$ das amostras capaz de reduzir o DPPH em 50\% em $30 \mathrm{~min}$.

\section{RESULTADOS E DISCUSSÃO}

\section{Composição química}

As estruturas das substâncias $\mathbf{1}$ a $\mathbf{8}$ isoladas das partes aéreas de Indigofera hirsuta foram identificadas a partir de dados espectroscópicos obtidos por RMN (mono- e bi-dimensionais) e comparadas com dados da literatura. ${ }^{27,28}$ Os espectros de RMN de ${ }^{1} \mathrm{He}$ de ${ }^{13} \mathrm{C}$ e os dados de UV e IV estão apresentados no material suplementar. Para confirmar a presença das substâncias nos respectivos ext. $\mathrm{MeOH}$ e extrato aquoso, as substâncias isoladas (1-6) e os extratos foram analisadas por CLAE-DAD-IES-EM (TOF). Esta análise permitiu combinar o tempo de retenção das substâncias com os dados de absorção no UV ${ }^{29,30}$ (Figuras 1a e 1b).

A técnica forneceu, também, informações sobre o íon molecular e fragmentos característicos que possibilitaram confirmar as estruturas das substâncias analisadas (Figura 2, Tabela 1). Os espectros de massas das substâncias 1 a $\mathbf{6}$ estão apresentados no material suplementar.

$\mathrm{O}$ extrato $\mathrm{MeOH}$ é constituído por flavonoides ( 3 e 4) e substâncias fenólicas (5 e 6), enquanto que o extrato aquoso contém as substâncias nitrogenadas triptofano (1) e uracila (2). (Tabela 1, Figura 2).

$\mathrm{O}$ aminoácido triptofano (1) com $\mathrm{t}_{\mathrm{r}}$ de $20 \mathrm{~min}$, exibiu o íon molecular protonado $[\mathrm{M}+\mathrm{H}]^{+}$em $\mathrm{m} / z, 205\left(\mathrm{C}_{11} \mathrm{O}_{2} \mathrm{H}_{13} \mathrm{~N}_{2}\right)$. A fragmentação de segunda ordem (EM/EM) de $\mathrm{m} / z, 205$ gerou os íons em $\mathrm{m} / \mathrm{z}, 188$, referente à perda de $\mathrm{NH}_{3}$ (fragmento $\mathrm{C}_{11} \mathrm{O}_{2} \mathrm{H}_{10} \mathrm{~N}$ ), e $\mathrm{m} / z, 146$, referente ao fragmento $\mathrm{C}_{9} \mathrm{OH}_{8} \mathrm{~N}$.

A uracila (2) com $\mathrm{t}_{\mathrm{r}}$ de 4 min apresentou íon molecular protonado $[\mathrm{M}+\mathrm{H}]^{+} \mathrm{em} \mathrm{m} / \mathrm{z}, 113$, de fórmula molecular $\left(\mathrm{C}_{4} \mathrm{O}_{2} \mathrm{H}_{5} \mathrm{~N}_{2}\right)$.

A rutina $(3)\left(\mathrm{t}_{\mathrm{r}}=31 \mathrm{~min}\right)$ apresentou o íon molecular protonado $[\mathrm{M}+\mathrm{H}]^{+}$em $\mathrm{m} / \mathrm{z}$ 611, indicando fórmula molecular $\mathrm{C}_{27} \mathrm{O}_{16} \mathrm{H}_{31}$. A fragmentação EM/EM gerou fragmentos representativos de $\mathrm{m} / z, 465$ $[\mathrm{M}-146+\mathrm{H}]^{+}$e de $m / z 303[\mathrm{M}-146-162+\mathrm{H}]^{+}$, indicando as perdas de ramnose (146) e glicose (162), respectivamente.

O kaempferol-3-O- $\beta$-D-glucopiranosídeo (4) ( $\left.\mathrm{t}_{\mathrm{r}}=34 \mathrm{~min}\right)$ apresentou o íon molecular protonado $[\mathrm{M}+\mathrm{H}]^{+} \mathrm{em} \mathrm{m} / \mathrm{z} 449$. A perda de uma unidade de glicose $[\mathrm{M}-162+\mathrm{H}]^{+}$levou à formação do fragmento referente à aglicona protonada $(\mathrm{m} / \mathrm{z}, 287)$. 
a)
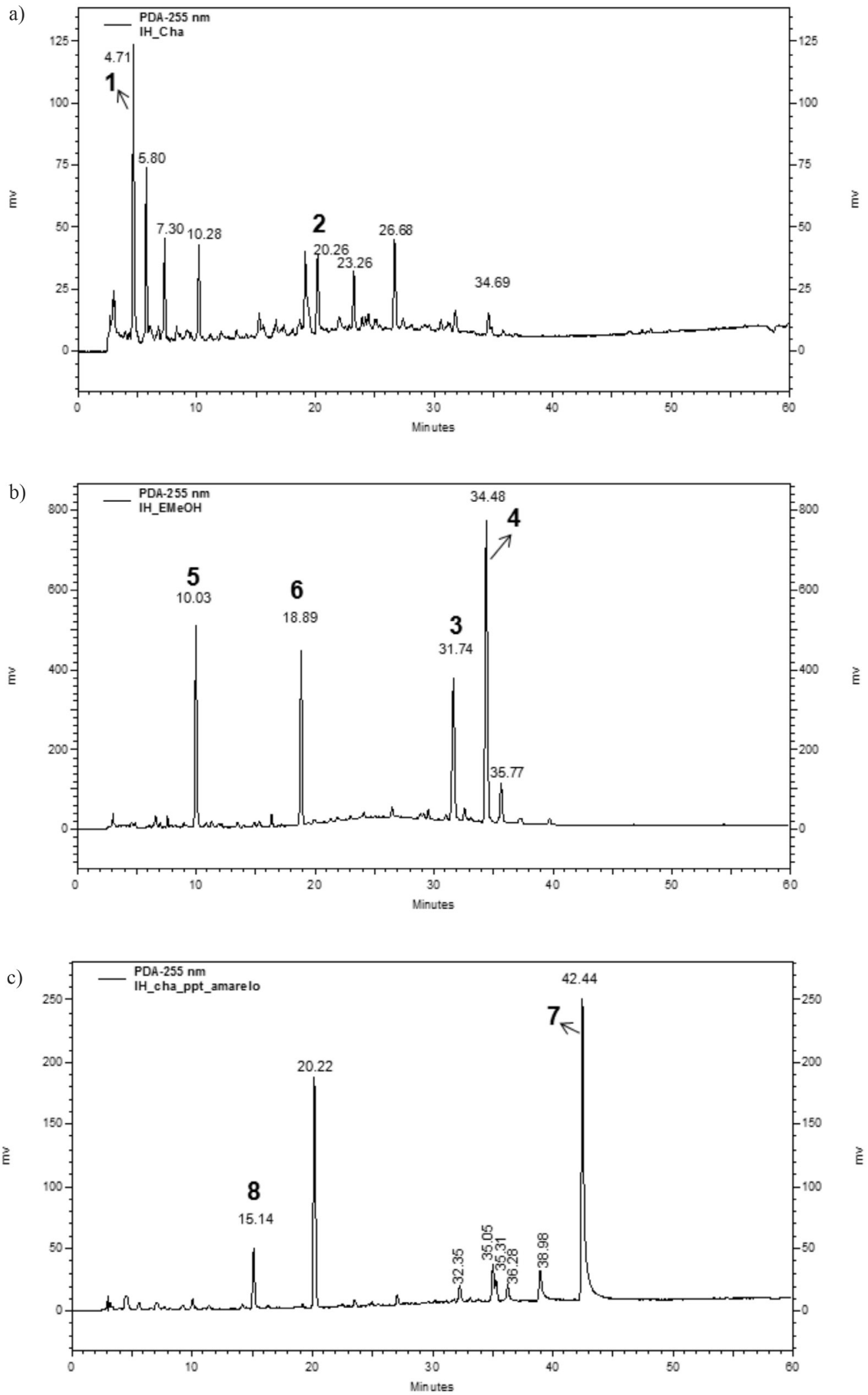

Figura 1. a) Cromatograma obtido por CLAE-DAD do extrato aquoso [CLAE-DAD (Jasco $\left.{ }^{\circledR}\right)$, coluna Luna 2 (Phenomenex $\left.{ }^{\circledR}\right) 5 \mu m(250 \times 4,60$ mm d.i), 254

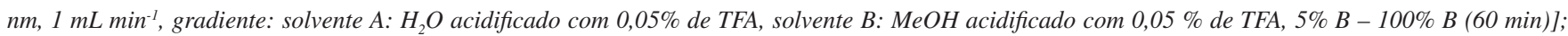
b) cromatograma obtido por CLAE-DAD do ext. MeOH [CLAE-DAD (Jasco $\left.{ }^{\oplus}\right)$, coluna Luna 2 (Phenomenex $\left.{ }^{\oplus}\right) 5 \mu \mathrm{m}\left(250 \times 4,60 \mathrm{~mm}\right.$ d.i), $254 \mathrm{~nm}, 1 \mathrm{~mL} \mathrm{~min}{ }^{-1}$, gradiente: solvente A: $\mathrm{H}_{2} \mathrm{O}$ acidificado com 0,05\% de TFA, solvente B: MeOH acidificado com 0,05\% de TFA, 5\% B-100\% B (60 min)]; c) cromatograma obtido por CLAE-DAD do precipitado [CLAE-DAD (Jasco $\left.{ }^{\oplus}\right)$, coluna Luna 2 (Phenomenex $\left.{ }^{\oplus}\right) 5$ um $\left(250 x\right.$ 4,60 mm d.i), $254 \mathrm{~nm} 1 \mathrm{~mL} \mathrm{~min}^{-1}$, gradiente: solvente A: $\mathrm{H}_{2} \mathrm{O}$ acidificado com 0,05\% de TFA, solvente B: $\mathrm{MeOH}$ acidificado com 0,05\% de TFA, 5\% B-100\% B (60 min)] 
Tabela 1. Dados das substâncias identificadas em Indigofera hirsuta

\begin{tabular}{|c|c|c|c|c|c|}
\hline Substância & Extrato & $\begin{array}{c}\mathrm{t}_{\mathrm{r}} \\
(\mathrm{min})\end{array}$ & $\begin{array}{c}\mathrm{UV} \\
\lambda_{\max }(\mathrm{nm})\end{array}$ & $\begin{array}{l}\text { Íon precursor } \\
\left([\mathrm{M}+\mathrm{H}]^{+}\right) \mathrm{m} / z\end{array}$ & $\begin{array}{l}\text { Íons produtos } \\
\text { (EM/EM) } \mathrm{m} / \mathrm{z}\end{array}$ \\
\hline Triptofano (1) & aquoso & 20 & 278 & 205 & $188 ; 146$ \\
\hline Uracila (2) & aquoso & 4 & 259 & 113 & - \\
\hline Rutina (3) & $\mathrm{MeOH}$ & 31 & $257 ; 356$ & 611 & $465 ; 303$ \\
\hline Kaempferol-3-O- $\beta$-D-glucopiranosídeo (4) & $\mathrm{MeOH}$ & 34 & $265 ; 347$ & 449 & 287 \\
\hline Ácido gálico (5) & $\mathrm{MeOH}$ & 9 & 273 & 171 & $153 ; 127 ; 109$ \\
\hline Galato de metila (6) & $\mathrm{MeOH}$ & 18 & 274 & 185 & $153 ; 126$ \\
\hline
\end{tabular}

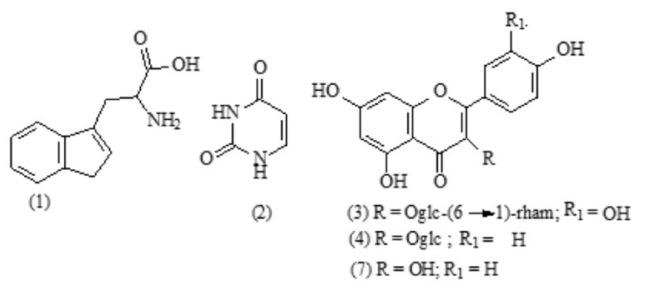<smiles>[R]C(=O)c1cc([R1])c(O)c(O)c1</smiles>

(5) $\mathrm{R}=\mathrm{OH} ; \mathrm{R}_{1}=\mathrm{OH}$ (6) $\mathrm{R}=\mathrm{OCH}_{3}: \mathrm{R}_{1}=\mathrm{OH}$ (8) $\mathrm{R}=\mathrm{OH}: \mathrm{R}_{1}=\mathrm{H}$

Figura 2. Substâncias identificadas nos ext. $\mathrm{MeOH}$, no extrato aquoso e no precipitado de Indigofera hirsuta

O espectro de massas do ácido gálico $(5)\left(\mathrm{t}_{\mathrm{r}}=9 \mathrm{~min}\right)$ exibiu o íon molecular protonado $[\mathrm{M}+\mathrm{H}]^{+} \mathrm{em} \mathrm{m} / z, 171$, indicando a fórmula molecular $\mathrm{C}_{7} \mathrm{O}_{5} \mathrm{H}_{7}$. A fragmentação de segunda ordem desse íon precursor levou aos fragmentos de $m / z, 153$ (perda de $\mathrm{H}_{2} \mathrm{O}[\mathrm{M}-18+\mathrm{H}]^{+}$ $\left.\left(\mathrm{C}_{7} \mathrm{O}_{4} \mathrm{H}_{5}\right)\right), \mathrm{m} / z, 127$ (perda de $\mathrm{CO}_{2}[\mathrm{M}-44+\mathrm{H}]^{+}\left(\mathrm{C}_{6} \mathrm{O}_{3} \mathrm{H}_{7}\right)$ ) e em $\mathrm{m} / z$ $109\left[\mathrm{C}_{6} \mathrm{O}_{3} \mathrm{H}_{7}-\mathrm{H}_{2} \mathrm{O}\right]^{+}$.

$\mathrm{O}$ galato de metila $(6)\left(\mathrm{t}_{\mathrm{r}}=18 \mathrm{~min}\right)$ exibiu o íon molecular protonado $[\mathrm{M}+\mathrm{H}]^{+} \mathrm{em} \mathrm{m} / \mathrm{z}, 185\left(\mathrm{C}_{8} \mathrm{O}_{5} \mathrm{H}_{9}\right)$. A fragmentação de segunda ordem gerou os íons de $m / z, 153\left(\mathrm{C}_{7} \mathrm{O}_{4} \mathrm{H}_{5}\right.$, perda da metoxila $\left.\left[\mathrm{M}-\mathrm{OCH}_{3}\right]^{+}\right) \mathrm{e}$ $m / z, 126\left(\mathrm{C}_{6} \mathrm{O}_{3} \mathrm{H}_{6}\right.$, perda de $\left.\mathrm{COOCH}_{3}\right)$.

A literatura reporta a existência de flavonoides em Indigofera hirsuta,${ }^{20}$ enquanto que as substâncias nitrogenadas triptofano (1) e uracila (2) bem como a rutina (3), o kaempferol-3-O- $\beta$-D-glucopiranosídeo (4), o ácido gálico (5), o galato de metila (6) o kaempferol (7) e o ácido protocatecuico $(\mathbf{8})$ estão sendo descritos pela primeira vez nesta espécie.

A identificação do triptofano tem grande importância, uma vez que está relacionado à via biossintética de alcaloides bis-indólicos como, por exemplo, o índigo e a indirubina. ${ }^{31,32}$ Estes alcaloides já foram identificados em algumas espécies do gênero Indigofera (I. suffruticosa e I. truxilelnsis), sendo obtidos dos extratos metanólicos dessas espécies. ${ }^{33}$

\section{Atividade antirradicalar}

As porcentagens de absorbância medidas pela equação $\left(\% \Delta_{0}\right)=$ $100 \times\left(\mathrm{A}_{0}-\mathrm{A}\right) / \mathrm{A}_{0}$ estão representadas na Figura 3 e os valores da $\mathrm{CE}_{50}$ na Tabela 2 .

Os resultados obtidos para o precipitado $\left(\mathrm{CE}_{50}=38,8 \pm 0,7\right)$, proveniente da centrifugação do extrato aquoso, e para o ext. $\mathrm{MeOH}$ $\left(\mathrm{CE}_{50}=67,7 \pm 0,9\right)$ contêm flavonoides e outras substâncias fenólicas e evidenciaram a atividade antirradicalar desses extratos.

O extrato aquoso $\left(\mathrm{CE}_{50}=462,7 \pm 1,0\right)$, que contém as substâncias nitrogenadas triptofano (1) e uracila (2), não apresentou atividade antirradicalar quando comparado aos demais extratos e ao padrão

Tabela 2. Concentração efeciente $\left(\mathrm{CE}_{50}\right)$ dos extratos e substâncias de Indigofera hirsuta

\begin{tabular}{lc}
\hline Amostras & $\mathrm{CE}_{50}\left(\mu \mathrm{g} \mathrm{mL}{ }^{-1}\right)$ \\
\hline Ácido gálico comercial $\left(\mathrm{Sigma}^{\oplus}\right)$ & $5 \pm 0,2^{*}$ \\
Precipitado & $38,8 \pm 0,7$ \\
Ext.MeOH & $67,7 \pm 0,9$ \\
Extrato aquoso & $462,7 \pm 1,0$ \\
Triptofano (1) & $186,1 \pm 1,0$ \\
Uracila (2) & $1958,0 \pm 0,9$ \\
Rutina (3) & $21,6 \pm 0.6$ \\
Kaempferol-3-O- $\beta$-D-glucopiranosídeo (4) & $457,8 \pm 1,0$ \\
Ácido gálico (5) & $5 \pm 0,2^{*}$ \\
Galato de metila $(\mathbf{6})$ & $5 \pm 0,3 *$ \\
Kaempferol (7) & $27,9 \pm 0,6$ \\
Ácido protocatecuico $(\mathbf{8})$ & $192,2 \pm 0,9$ \\
\hline
\end{tabular}

*O ácido gálico padrão e as substâncias 5 e $\mathbf{6}$ reduziram o DPPH em mais de $50 \%$ na menor concentração analisada $\left(5 \mu \mathrm{g} \mathrm{mL}^{-1}\right)$.

\section{Atividade antirradicalar com DPPH}

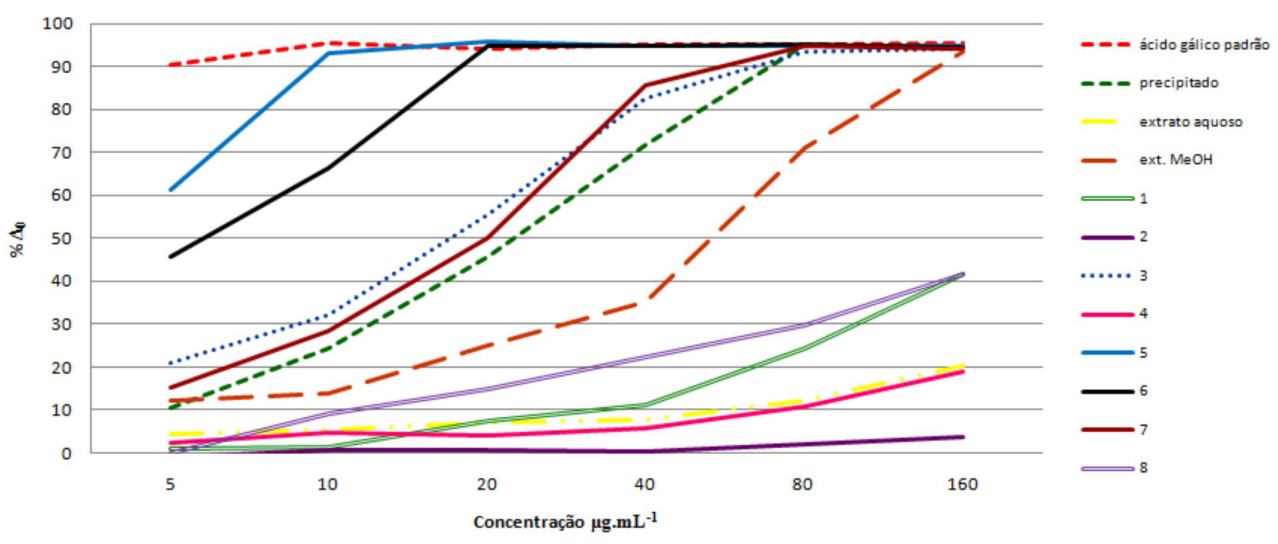

Figura 3. Ensaio antirradicalar do ácido gálico padrão, precipitado, extrato aquoso, ext. MeOH e das substânicas isoladas (1-8) de Indigofera hirsuta 
do ácido gálico comercial $\left(\mathrm{CE}_{50}=5 \pm 0,2\right)$.

$\mathrm{O}$ ácido gálico (5) $\left(\mathrm{CE}_{50}=5 \pm 0,2\right)$ isolado do ext. $\mathrm{MeOH}$ e o galato de metila (6) $\left(\mathrm{CE}_{50}=5 \pm 0,3\right)$ foram as substâncias que, na menor concentração, reduziram o DPPH em mais de $50 \%$, enquanto que o ácido protocatecuido $(\mathbf{8})$ apresentou $\mathrm{CE}_{50} 192,2 \pm 0$,9. Esse fato pode ser explicado pela presença de duas hidroxilas fenólicas em 8 e três em 5 e $6^{26,34}$

A rutina (3) apresentou $\mathrm{CE}_{50}=21,6 \pm 0,6$, enquanto que o kaempferol-3-O- $\beta$-D-glucopiranosídeo (4) exibiu $\mathrm{CE}_{50}=457,8 \pm 1,0$. A menor atividade antirradicalar de $\mathbf{4}$ em comparação à rutina (3) é justificada devido à existência do sistema orto-di-hidroxi no anel $\mathrm{B}$ de $\mathbf{3}^{34}$

O kaempferol (7) $\left(\mathrm{CE}_{50}=27,9 \pm 0,6\right)$ não possui o sistema orto-dihidroxi no anel B sendo, portanto, sua atividade antirradicalar menos pronunciada que em 3. A menor atividade antirradicalar da substância 4, um derivado do kaempferol, $\left(\mathrm{CE}_{50}=457,8 \pm 1,0\right)$, em comparação com 7, é justificada pela presença da glicose na posição 3 de $4 .{ }^{34}$

Comparadas aos flavonoides as substâncias 1 e 2 não têm a propriedade de estabilizar o radical formado, pois não apresentam em suas estruturas hidroxilas fenólicas (Figura 2), sendo, portanto, pouco efetiva a ação de sequestrar o hidrogênio radicalar para formar a difenilpicrilhidrazina. ${ }^{35,36}$

Os flavonoides já são considerados substâncias com potentes propriedades antirradicalares. ${ }^{35,36}$ A presença de flavonoides $(\mathbf{3}, \mathbf{4}$ e 7) e outros fenólicos $(\mathbf{5}, \mathbf{6}$ e $\mathbf{8})$ no precipitado e no ext. $\mathrm{MeOH}$ das partes aéreas de Indigofera hirsuta justifica a propriedade antirradicalar da espécie.

\section{CONCLUSÃO}

A técnica CLAE-IES-EM (TOF) mostrou-se adequada para identificar os constituintes químicos dos ext. $\mathrm{MeOH}$ e aquoso das partes aéreas de Indigofera hirsuta. Através da análise dos íons moleculares protonados e dos fragmentos nos espectros de massa foi possível identificar as substâncias isoladas dos extratos como sendo: triptofano (1), uracila (2), rutina (3), kaempferol-3-O- $\beta$-D-glucopiranosideo (4), ácido gálico (5) e galato de metila (6). As substâncias kaempferol (7) e ácido protocatecuico (8) foram isolados do precipitado (Figura 1c).

Comparando-se os extratos de Indigofera hirsuta, a melhor atividade sequestradora de radicais livres foi obtida para o precipitado, seguido do extrato metanólico e do extrato aquoso. Esta atividade é justificada pela presença do kaempferol no precipitado, da rutina, ácido gálico e galato de metila, no ext. MeOH. Essas são substâncias capazes de melhor sequestrar um hidrogênio radicalar do DPPH e formar um radical estável.

\section{MATERIAL SUPLEMENTAR}

Os espectros de massas, os espectros de $\mathrm{RMN}^{1} \mathrm{H}$, os dados de $\mathrm{RMN}$ de ${ }^{13} \mathrm{C}$, UV e IV encontram-se disponíveis gratutitamente em http:// quimicanova.sbq.org.br, na forma de arquivo PDF.

\section{AGRADECIMENTOS}

Ao CNPq, à CAPES e FAPESP pelo apoio financeiro. À Profa. Dra. V. da S. Bolzani (NUBBE, IQ-UNESP-Araraquara), ao Prof. Dr. N. P. Lopes (USP-RP) pela colaboração na realização dos experimentos de HPLC-ESI-MS (TOF) e ao técnico J. C. Tomaz (USP-RP) pelo registro dos espectros de massas.

\section{REFERÊNCIAS}

1. Sultana, B.; Anwar, F.; Food Chem. 2008, 108, 879.

2. Li, H. B.; Wong, C.; Cheng, K.; Chen, F.; Food Sci. Technol. 2008, 41, 385 .
3. Barroso, G, M.; Sistemática de angiospermas do Brasil, $2^{\text {a }}$ ed., Edusp: São Paulo, 1978.

4. Di Stasi, L. C.; Hiruma-Lima, C. A.; Plantas medicinais na Amazônia e na Mata Atlântica, 2a ed., Ed. UNESP: São Paulo, 2002.

5. Moreira, J. L. A.; Azevedo-Tozzi, A. N. G.; Rev. Bras. Bot. 1997, $20,97$.

6. Barros, G. M. C. C.; Teixeira, S. P.; Rev. Bras. Farmacogn. 2008, 18, 287.

7. Hasan, A.; Farman, M.; Ahmad, I.; Phytochemistry 1994, 35, 275.

8. Hasan, A.; Ahmad, I.; Khan, M. A.; Chudhary, M. I.; Phytochemistry 1996, 43, 1115

9. Kamal, R.; Mangla, M.; Biol. Plant. 1984, 263, 202.

10. Adinarayana, D.; Sarada, M. J.; Indian Chem. Soc. 1987, 64, 648.

11. Anila, T.; Nancy, R.; Ahmed, S. R. J.; Indian Chem. Soc. 1982, 59, 1007.

12. Hasan, A.; Khan, M. A.; Iftikhar, N.; Fitoterapia 1991, 52, 183.

13. Hasan, A.; Waterman, P.; Iftikhar, N.; J. Chromatogr. 1989, 466, 399.

14. Moraes e Souza, M. A.; Bieber, L. W.; Chiappeta, A. A.; Maciel, G. M.; Mello, J. F.; Monache, F. D.; Messana, I.; Phytochemistry 1988, 27, 1817.

15. Williams, M. C.; Agron. J. 1981, 73, 434.

16. Aylward, J. H.; Court, R. D.; Haydock, K. P.; Strickland, R. W.; Hegarty, M. P.; Aust. J. Agric. Res. 1987, 38, 177.

17. Dominguez, X. A.; Martinez, C.; Calero, A.; Dominguez-Jr, X. A.; Hinojosa, M.; Samudio, A.; Watson, W. H.; Zabel, U.; Planta Med. 1978, 34,172 .

18. Dominguez, X. A.; Martinez, C.; Calero, A.; Dominguez-Jr, X. A.; Hinojosa, M.; Zamudio, A.; Zabel, U.; Smith, W. B.; Watson, W. H.; Tetrahedron Lett. 1978, 5, 429.

19. Garcez, W. S.; Garcez, F. R.; Honda, N. K.; Ciência Cult. (Suppl.) 1985, 37,312 .

20. Rao, J. U. M.; Hanumiah, T.; Rao, B. K.; Rao, K. V. J.; Indian J. Chem., Sect. B: Org. Chem. Incl. Med. Chem. 1984, 23, 91.

21. Morris, J. B.; Walker, J. T.; J. Nemat. 2002, 34, 358

22. Wagner, H.; Bladt, S.; Zgainski, E. M.; Plant Drug Analysis, Springer Verlag: Berlin, 1984.

23. Molyneux, P.; Songklanakarin J. Sci. Technol. 2004, 26, 211.

24. Silva, M. A.; Tese de Doutorado, Universidade Estadual Paulista, Brasil, 2008.

25. Jang, H. D.; Chang, K. S.; Chang, T. C.; Hsu, C. L.; Food Chem. 2010, $118,554$.

26. Sousa, C. M. M.; Silva, H. R.; Vieria-Jr., G. M.; Ayres, M. C. C.; Costa, C. L. S.; Araújo, D. S.; Cavalvante, L. C. D.; Barros, E. D. S.; Araújo, P. B. M.; Brandão, M. S.; Chaves, M. H.; Quim. Nova 2007, 30, 351.

27. Agrawal, P. K.; Carbon 13 NMR of flavonoids, Elsevier: Amsterdam, 1989.

28. Harborne, J. B.; The flavonoids: advances in research since 1986, Chapman and Hall: London, 1996.

29. Mabry, T. J.; Markham, K. R.; Thomas, M. B.; The systematic identification of flavonoids, Springer-Verlag: New York, 1970.

30. Rinaldo, D.; Dissertação de Mestrado, Universidade Estadual Paulista, Brasil, 2007.

31. Salvini, M.; Boccardi, T. M.; Sani, E.; Bernardi, R.; Tozzi, S.; Pugliesi, C.; Durante, M.; Plant Physiol. Biochem. 2008, 46, 715.

32. Berry, A.; Dodge, T. C.; Pepsin, M.; Weyler, W.; J. Ind. Microbiol. Biotechnol. 2002, 28, 127.

33. Calvo, T. R.; Cardoso, C. R. P.; Moura, A. C. S.; Santos, L. C.; Colus, I. M. S.; Vilegas, W.; Varanda, E. A.; Evid. Based Complement. Altern. Med. (2009), doi: doi:10.1093/ecam/nep123.

34. Ayres, M. C. C.; Chaves, M. H.; Rinaldo, D.; Vilegas, W.; Vieira-Jr., G. M.; Quim. Nova 2009, 32, 1509.

35. van den Ber, R.; Haenen, G. R. M. M.; van den Berg, H.; Vander, V. W.; Bast, A.; Food Chem. 2000, 703, 391.

36. Saija, A.; Scalese, M.; Lanza, M.; Marzullo, D.; Bonina, F.; Castelli, F.; Free Radical Biol. Med. 1995, 4, 481. 
IDENTIFICAÇÃO DE ALGUNS CONSTITUINTES QUÍMICOS DE Indigofera hirsuta LINN. (FABACEAE) POR CLAE-IES-EM (TOF) E AVALIAÇÃO DA ATIVIDADE ANTIRRADICALAR

Adriana Candido da Silva Moura*, Wagner Vilegas e Lourdes Campaner dos Santos

Departamento de Química Orgânica, Instituto de Química, Universidade Estadual Paulista, CP 355, 14800-900 Araraquara - SP, Brasil

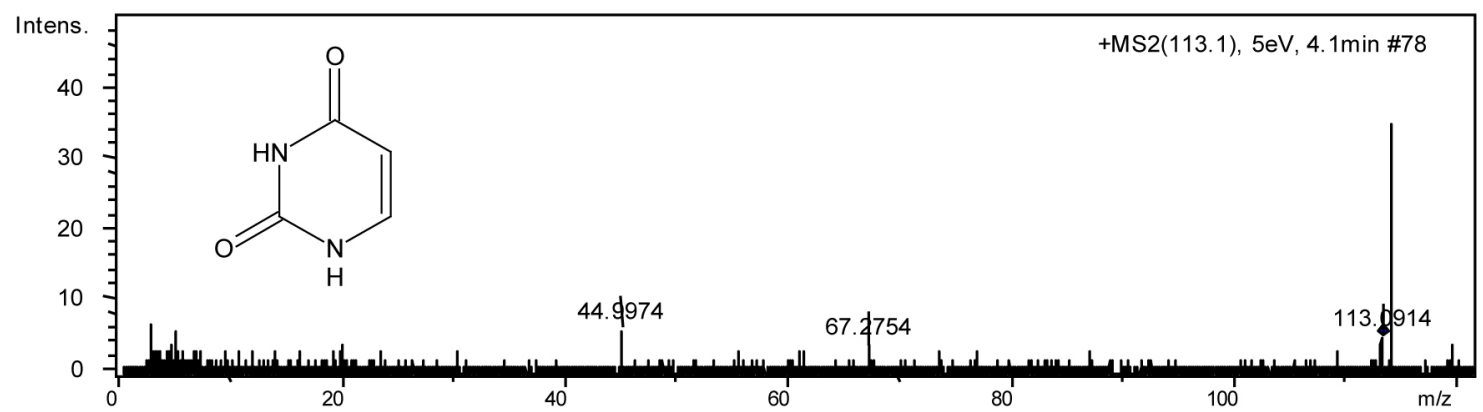

Figura 1S. Espectro de massas (EM/EM) da uracila (1)

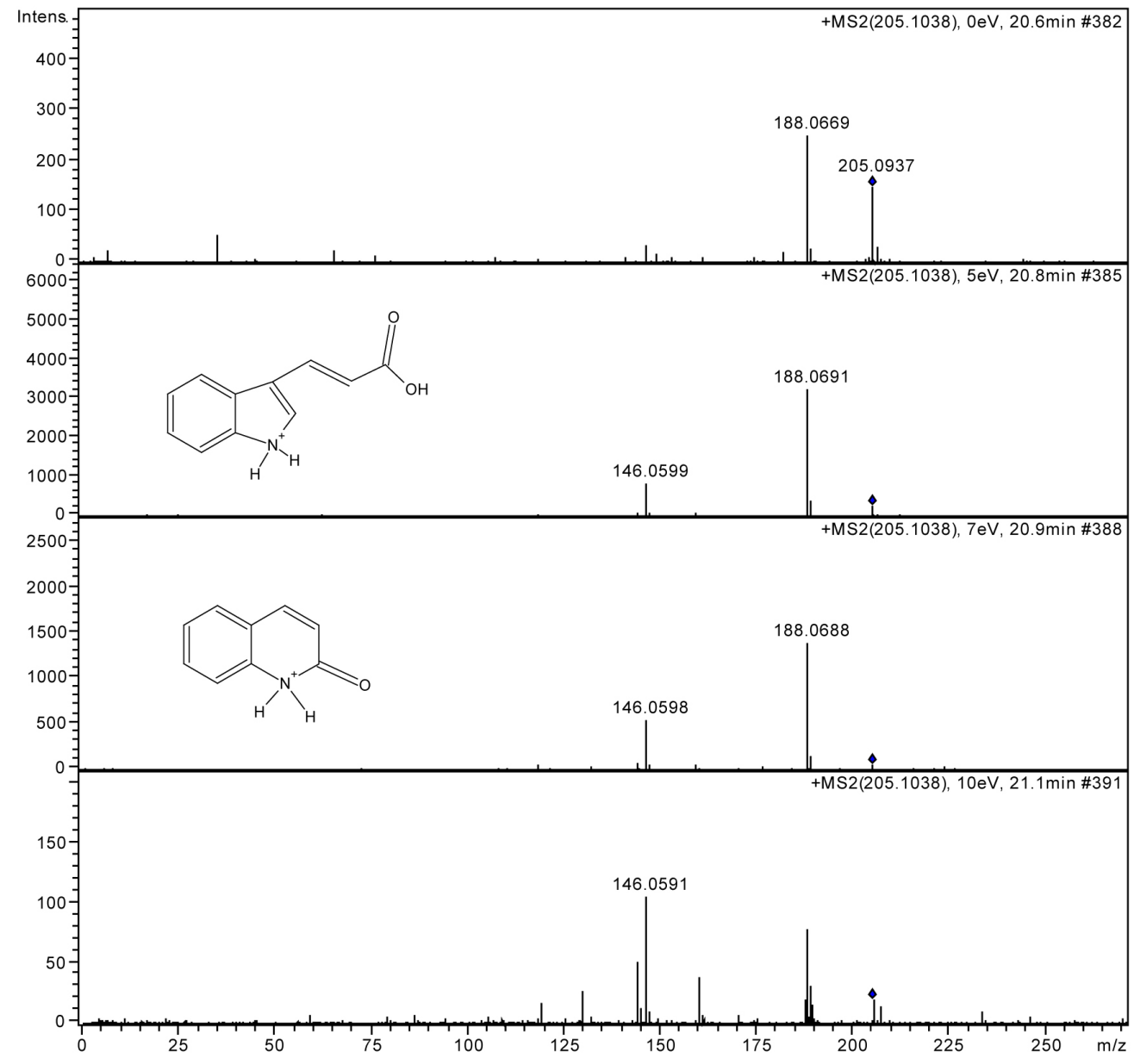

Figura 2S. Espectro de massas (EM/EM) de triptofano (2) em diferentes energias (0, 5, 7 e $10 \mathrm{eV})$ 


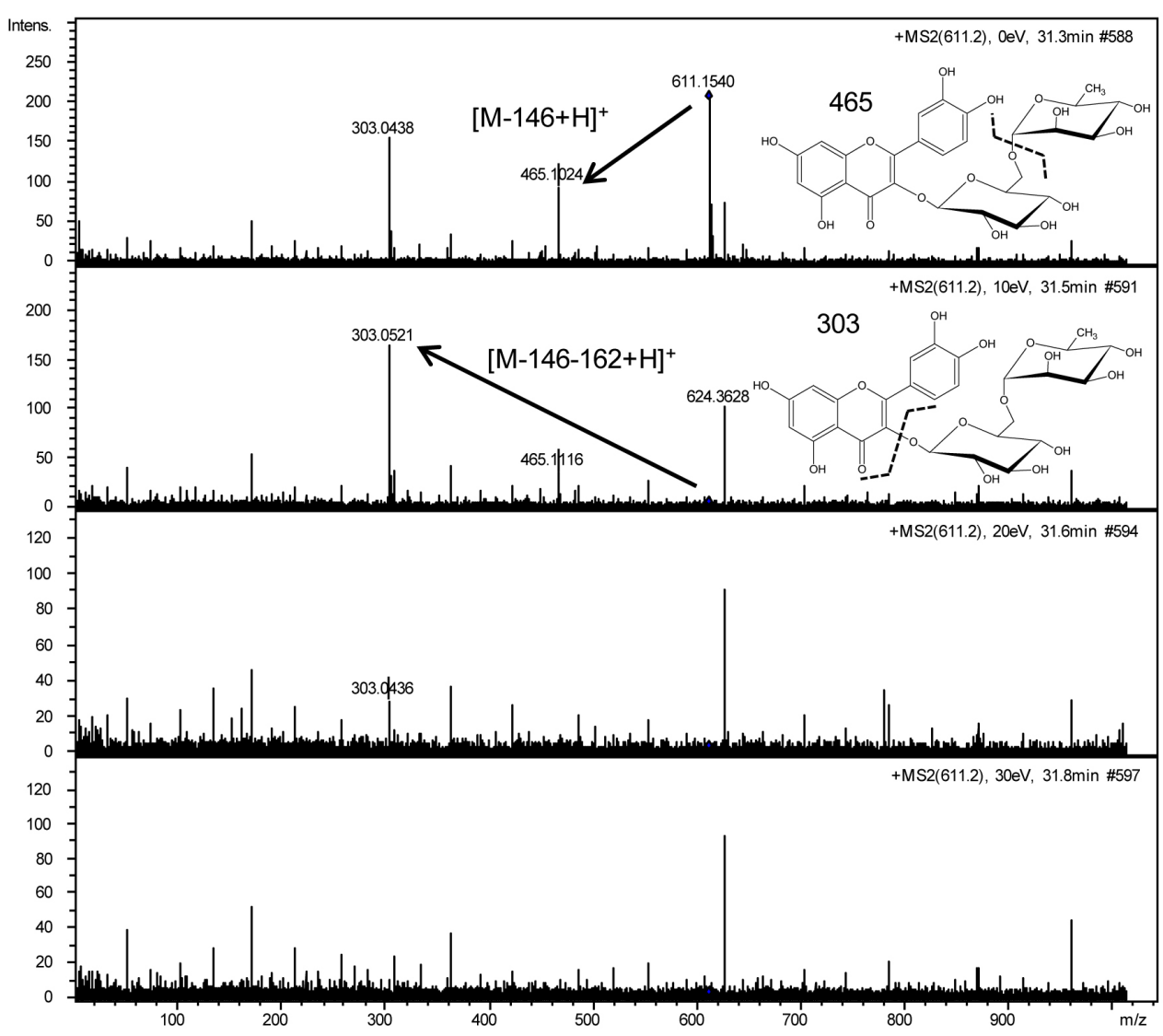

Figura 3S. Espectro de massas (EM/EM) da rutina (3) em diferentes energias (0, 10, 20 e $30 \mathrm{eV})$

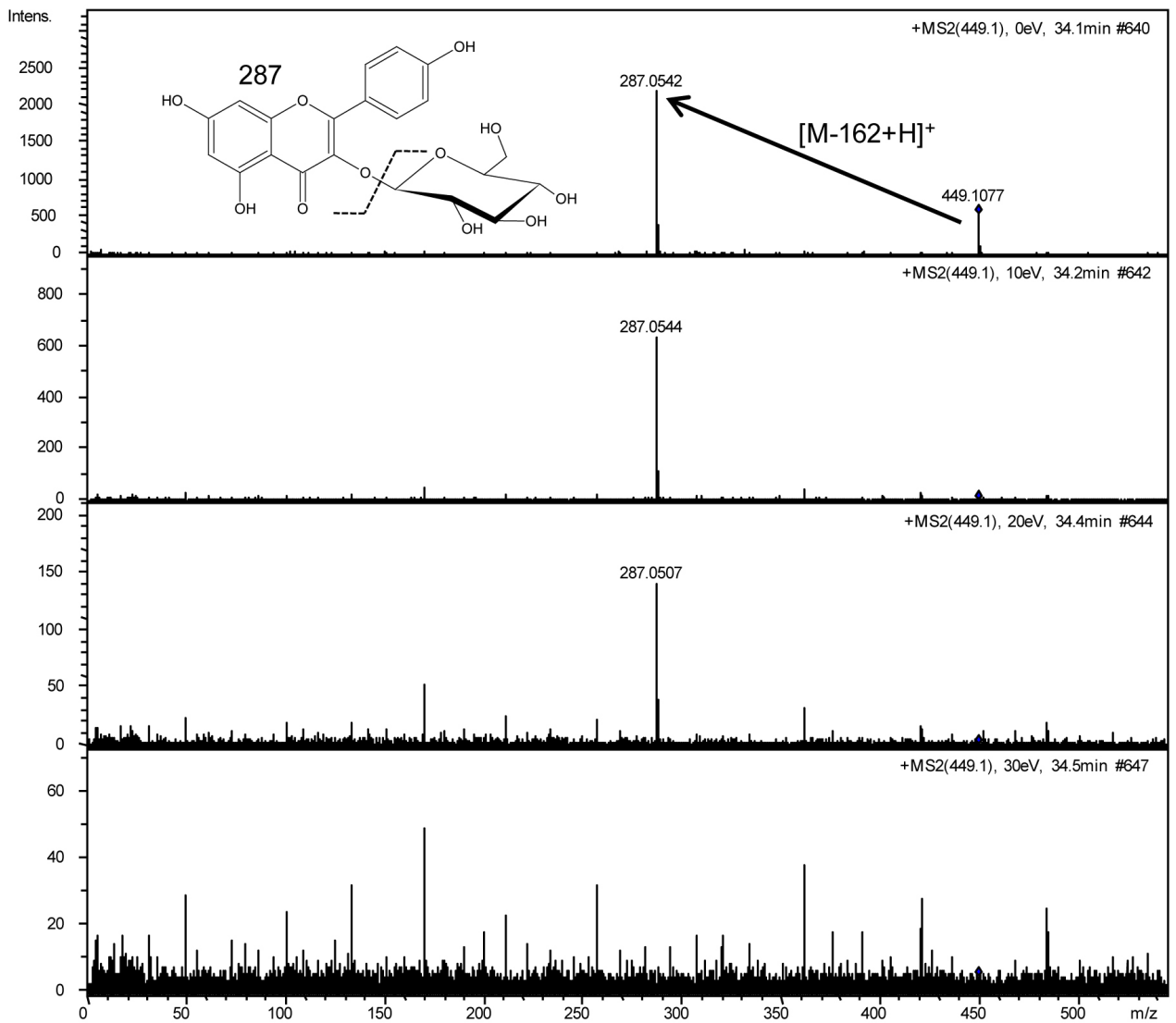

Figura 4S. Espectro de massas (EM/EM) do kaempferol-3-O-B-D-glucopiranosideo (4) em diferentes energias (0, 10, 20 e 30 eV) 


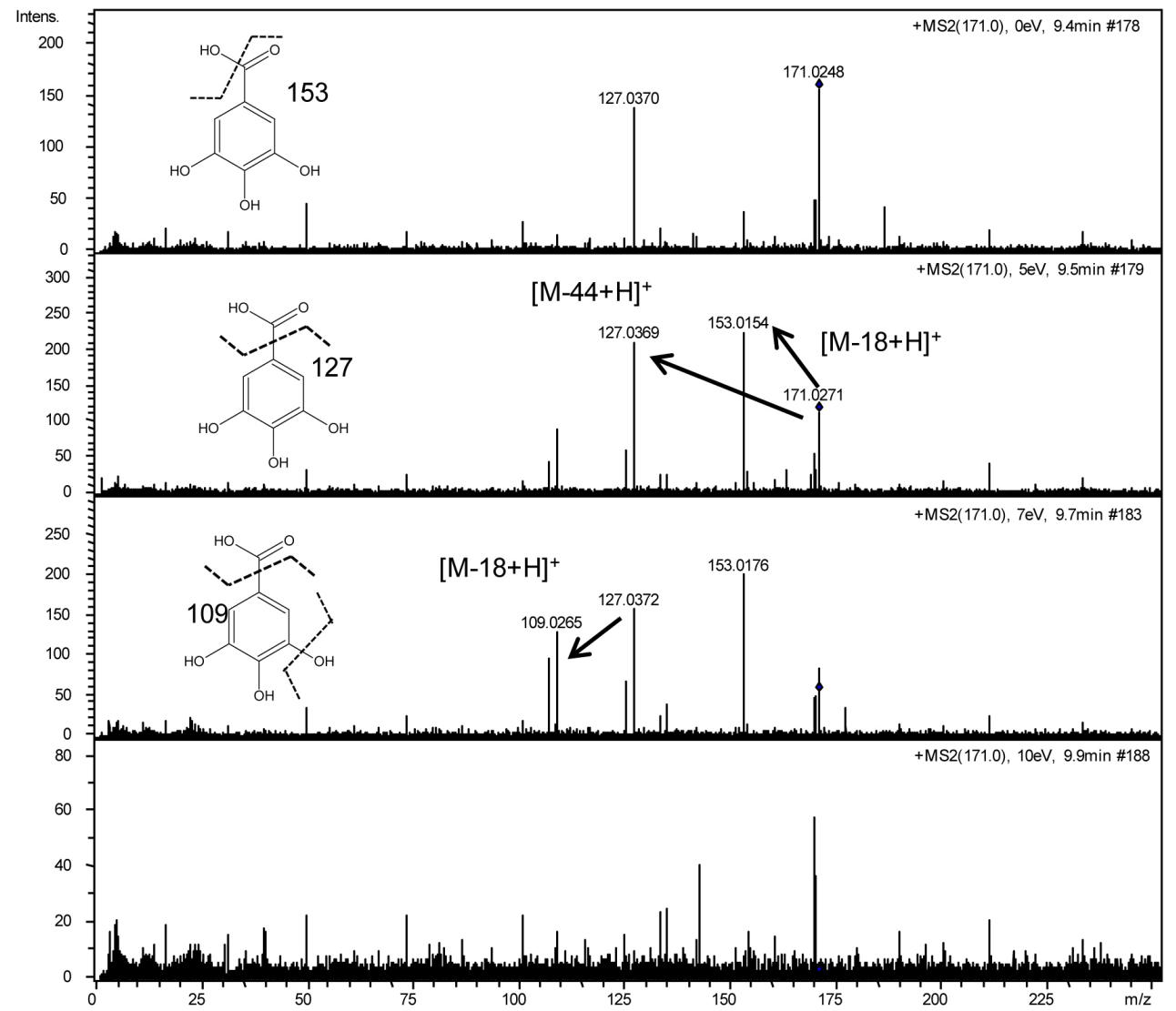

Figura 5S. Espectro de massas (EM/EM) do ácido gálico (5) em diferentes energias (0, 5, 7 e $10 \mathrm{eV})$

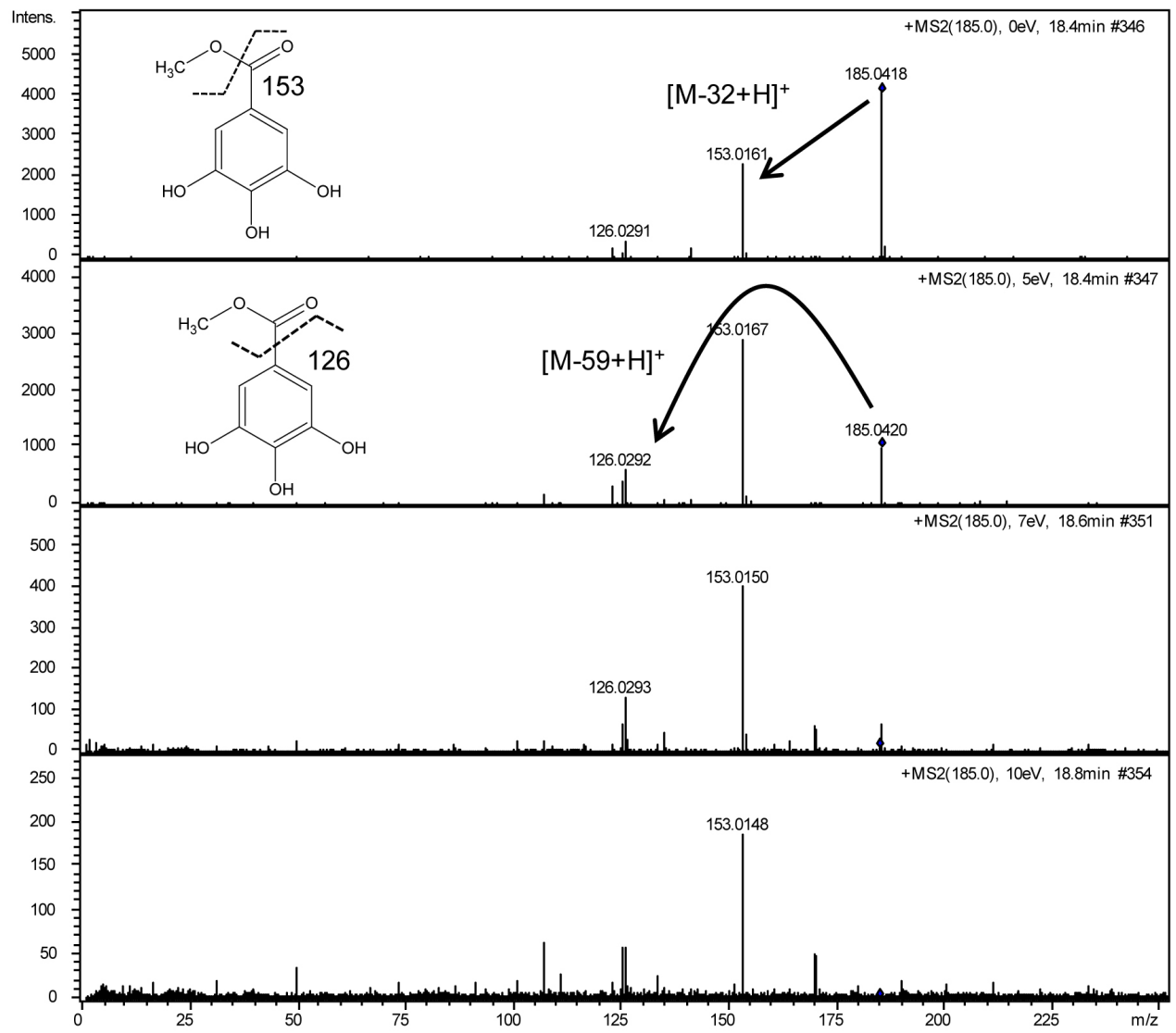

Figura 6S. Espectros de massas (EM/EM) do galato de metila (6) em diferentes energias (0, 5, 7 e $10 \mathrm{eV})$ 
Dados de RMN de ${ }^{1} \mathrm{H}$, de ${ }^{13} \mathrm{C}$ (obtidos através de experimentos gHMQC e gHMBC) e de UV e IV das substâncias (1-8):

Triptofano (1): RMN ${ }^{1} \mathrm{H}\left[500 \mathrm{MHz}\right.$, DMSO-d ${ }_{6}, \delta$ (ppm), J (Hz)]: 7,52 (s; H-2); 7,58 (d, J=8,0 Hz; H-4); 6,98 (ddd, J=8,0; J=8,0 e J=0,5 Hz; H-5); 7,07 (ddd, J=8,0; J=8,0 e J=0,5 Hz; H-6); 7,35 (d, J=8,0 Hz; H-7); 3,5 (m; H- $\alpha$ ); 3,32 e 2,99 (m; H- $\beta$ ). RMN ${ }^{13} \mathrm{C}\left[125 \mathrm{MHz}, \mathrm{DMSO}-\mathrm{d}_{6}, \delta\right.$ (ppm)]: 124,0 (C-2); 110,0 (C-3); 118,0 (C-4; C-5); 121,0 (C-6); 111,0 (C-7); 127,0 (C-3a); 136,0 (C-7a); 54,0 (C- $\alpha) ; 27,0$ (C- $\beta)$. UV: 278 nm. IV $\left(\mathrm{cm}^{-1}\right): v_{\mathrm{NH} 2}$ em 3288; $v_{\mathrm{C}-\mathrm{H}}$ em 2920 e $v_{\mathrm{CH}_{2}}$ em 2853; $\delta_{\mathrm{N}-\mathrm{H}(\text { ass })}$ em $1631 \mathrm{~cm} ; v_{\mathrm{C}=\mathrm{C}(\text { aromático) }}$ e $v_{\mathrm{C}=\mathrm{N}(\text { aromático) }}$ em $1602 ; v_{\mathrm{C}-\mathrm{O}}$ em $1402 ; v_{\mathrm{C}-\mathrm{N}}$ em 1091.

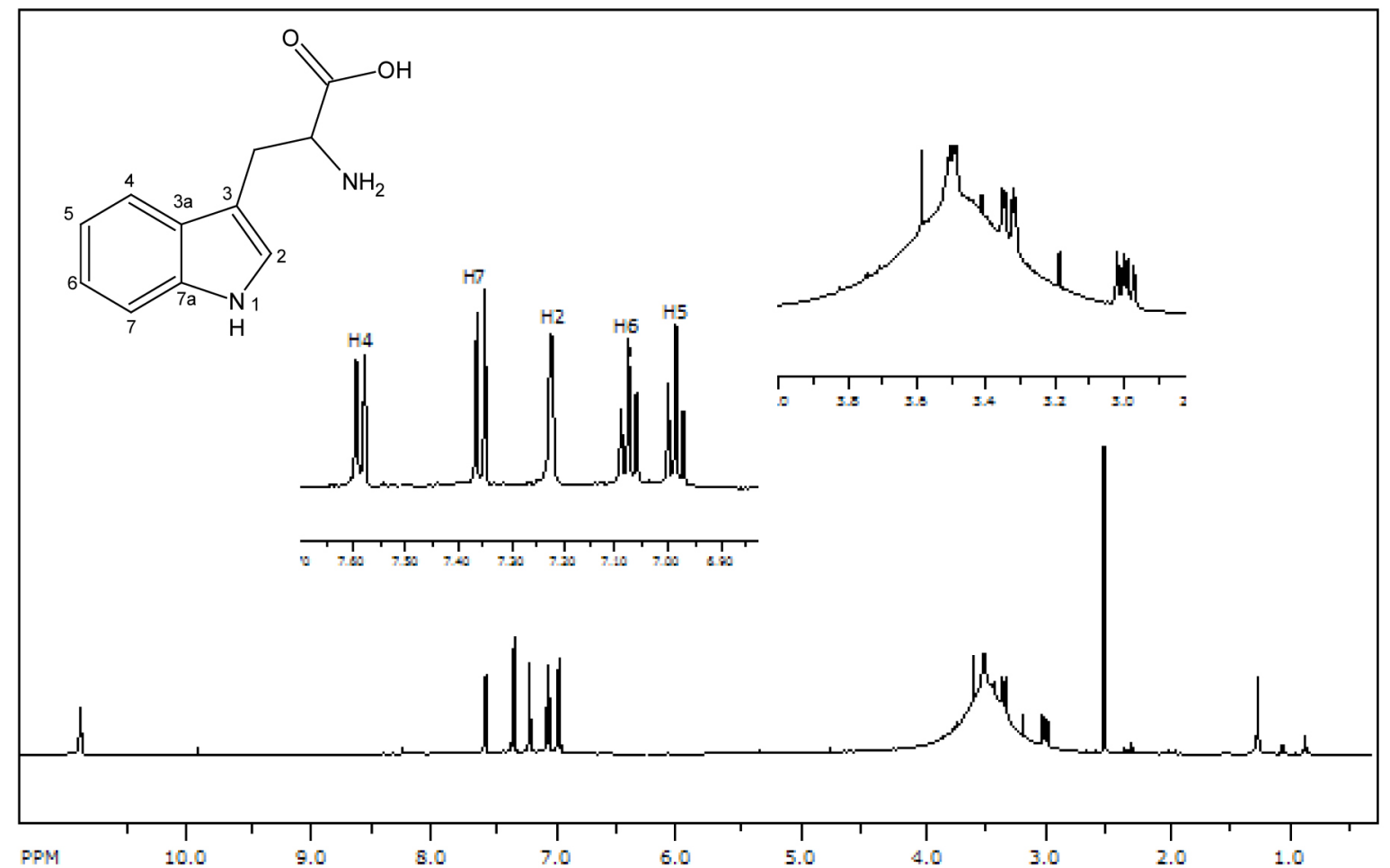

Figura 7S. Espectro de RMN de ${ }^{l} H$ do triptofano (1)

Uracila (2): RMN $\left.{ }^{1} \mathrm{H}\left[500 \mathrm{MHz}, \mathrm{DMSO}-\mathrm{d}_{6}, \delta(\mathrm{ppm}), \mathrm{J}(\mathrm{Hz})\right]:\right]: 5,45$ (d, J=7,5 Hz, H-5); 7,38 (d, J=7,5 Hz, H-6). RMN ${ }^{13} \mathrm{C}[125 \mathrm{MHz}$, DMSO-d $\left._{6}, \delta(\mathrm{ppm})\right]: 151,6(\mathrm{C}-2) ; 164,4(\mathrm{C}-4) ; 100,2$ (C-5); 142,3 (C-6). UV: $259 \mathrm{~nm}$. IV (cm-1): $v_{\mathrm{N}-\mathrm{H}}$ em 3370; $v_{\mathrm{C}=\mathrm{O}}$ em 1702; $\delta_{\mathrm{N}-\mathrm{H}}$ em 1578; $v_{\mathrm{C}-\mathrm{N}}$ em 1392.
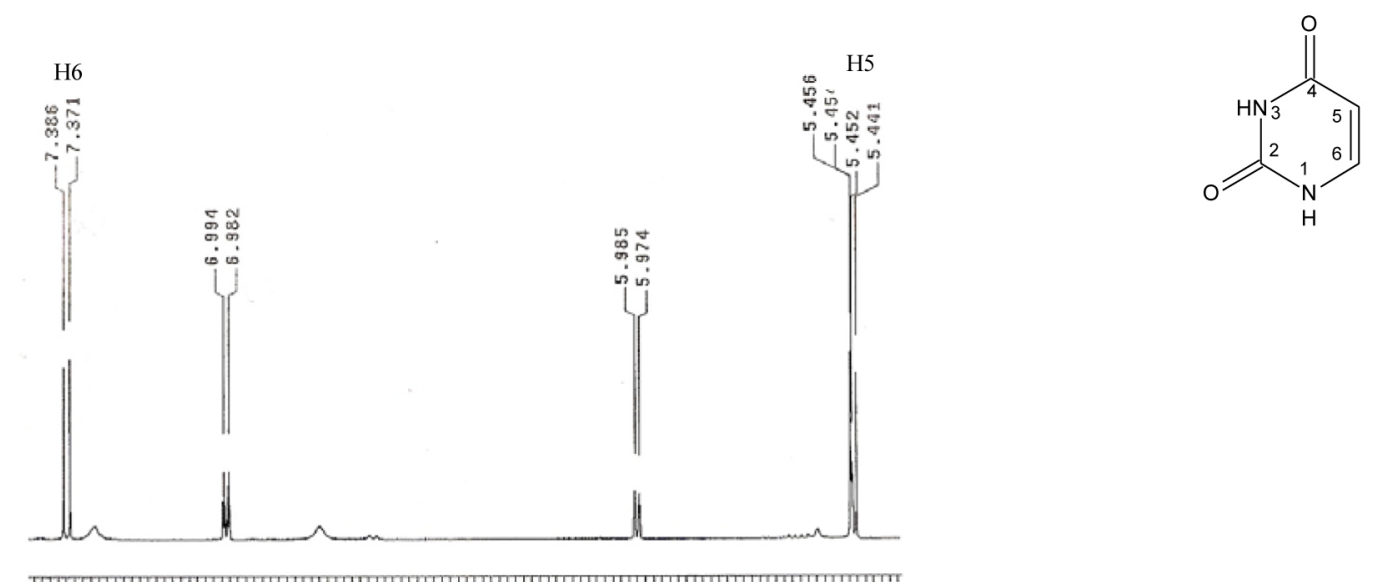

$\begin{array}{lllllllllll}7.4 & 7.2 & 7.0 & 6.8 & 6.6 & 6.4 & 6.2 & 6.0 & 5.8 & 5.6 & \mathrm{ppm}\end{array}$

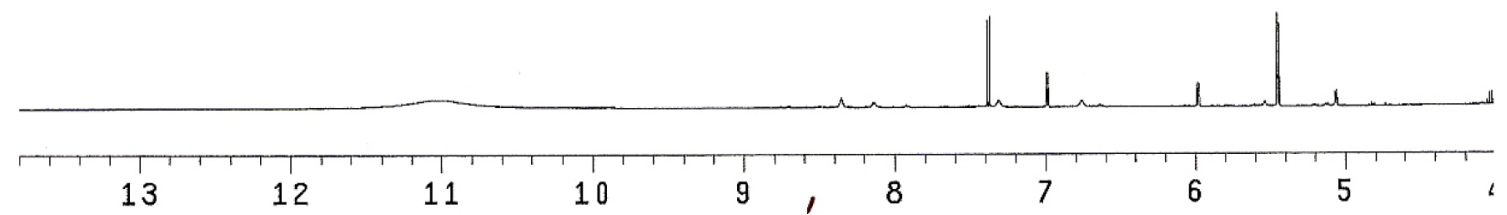

Figura 8S. Espectro de RMN de ${ }^{1} \mathrm{H}$ da uracila (2) 
Rutina (3): RMN ${ }^{1} \mathrm{H}\left[500 \mathrm{MHz}, \mathrm{DMSO}_{\mathrm{d}}, \delta\right.$ (ppm), J (Hz)]: 6,20 (d, J=2,1 Hz, H-6); 6,40 (d, J=2,1 Hz, H-8); 7,53 (d, J=2,0 Hz, H-2'); 6,83 (d, J=9,0 Hz, H-5'); 7,75 (dd, J=7,8 e J=2,4 Hz, H-6'); 5,44 (d, J=7,2 Hz, H-1 "); 4,38 (d, J=1,0 Hz, H-1'”); 0,99 (d, J=6,0 Hz, H-6"'). $\mathrm{RMN}^{13} \mathrm{C}\left[125 \mathrm{MHz}, \mathrm{DMSO}-\mathrm{d}_{6}, \delta(\mathrm{ppm})\right]: 156,5$ (C-2); 133,2 (C-3); 177,3 (C-4); 161,2 (C-5); 98,7 (C-6); 164,5 (C-7); 93,6 (C-8); 156,4 (C9); 103,7 (C-10); 121,5 (C-1'); 116,2 (C-2'); 115,2 (C-3'); 148,5 (C-4'); 115,2 (C-5'); 121,0 (C-6'); 101,2 (C-1'”); 75,8 (C-2'”); 74,0 (C-3'”); 69,9 (C-4"); 76,4 (C-5"); 66,9 (C-6"); 100,7 (C-1"”); 70,5 (C-2"’); 70,3 (C-3"”); 71,8 (C-4"”); 68,2 (C-5"'); 17,6 (C-6”"). UV: 258 nm e 354

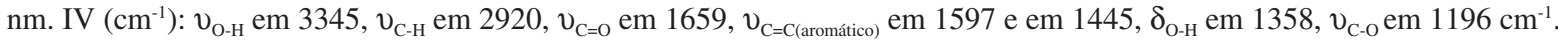
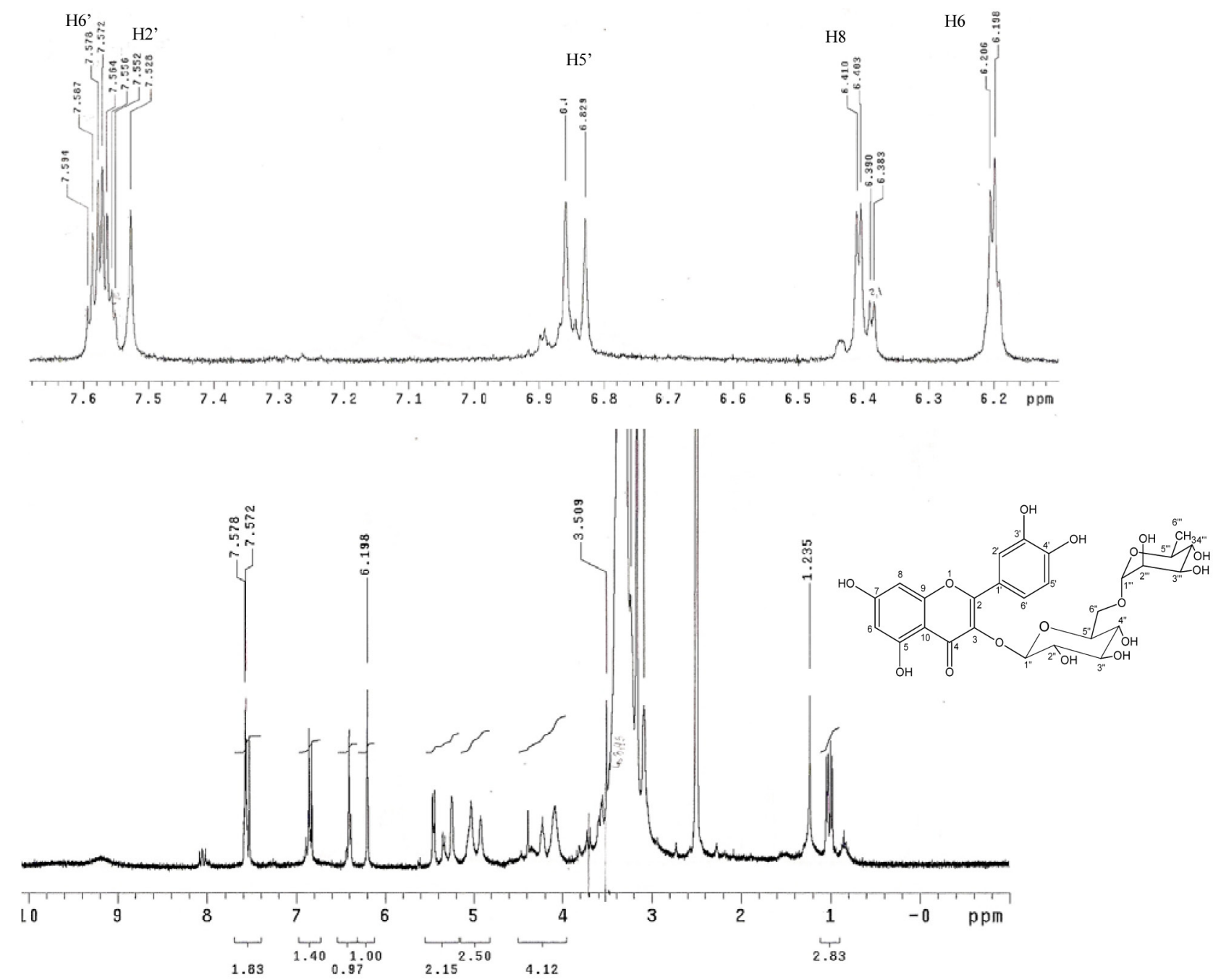

Figura 9S. Espectro de RMN de ${ }^{1} \mathrm{H}$ da rutina (3) 


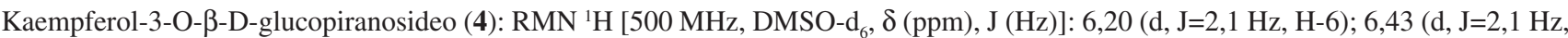
H-8); 7,97 (d, J=8,5 Hz, H-2'); 6, 84 (d, J=8,5 Hz, H-3'); 6,84 (d, J=8,5 Hz, H-5'); 7,97 (d, J=8,5 Hz, H-6'); 5,28 (d, J=7,5 Hz, H-1'); 3,18 (H-2"); 3,20 (H-3"); 3,10 (H-4”); 3,13 (H-5”); 3,55; 3,35 (H-6”). RMN ${ }^{13} \mathrm{C}$ [125 MHz, DMSO-d ,, (ppm)]: 156,2 (C-2); 133,2 (C-3); 177,4 (C-4); 161,2 (C-5); 98,6 (C-6); 164,1 (C-7); 93,6 (C-8); 156,4 (C-9); 104,0 (C-10); 120,8 (C-1'); 130,8 (C-2'); 115,1 (C-3'); 159,9 (C-4'); 115,1 (C-5'); 130,8 (C-6'); 100,8 (C-1”); 74,2 (C-2”); 76,4 (C-3”); 69,9 (C-4”); 77,4 (C-5”); 60,8 (C-6”). UV: 264 nm e 345 nm. IV (cm'): $v_{\mathrm{O}-\mathrm{H}}$ em 3242, $v_{\mathrm{C}=\mathrm{O}}$ em 1655, $v_{\mathrm{C}=\mathrm{C}(\text { aromático) }}$ em 1607 e em 1505, $\delta_{\mathrm{O}-\mathrm{H}}$ em 1354, $v_{\mathrm{C}-\mathrm{O}}$ em 1175, $\delta_{\mathrm{C}-\mathrm{H}(\text { aromático) }}$ em 840.<smiles>O=C(O)C(O)COc1c(-c2ccc(O)cc2)oc2cc(O)cc(O)c2c1=O</smiles>

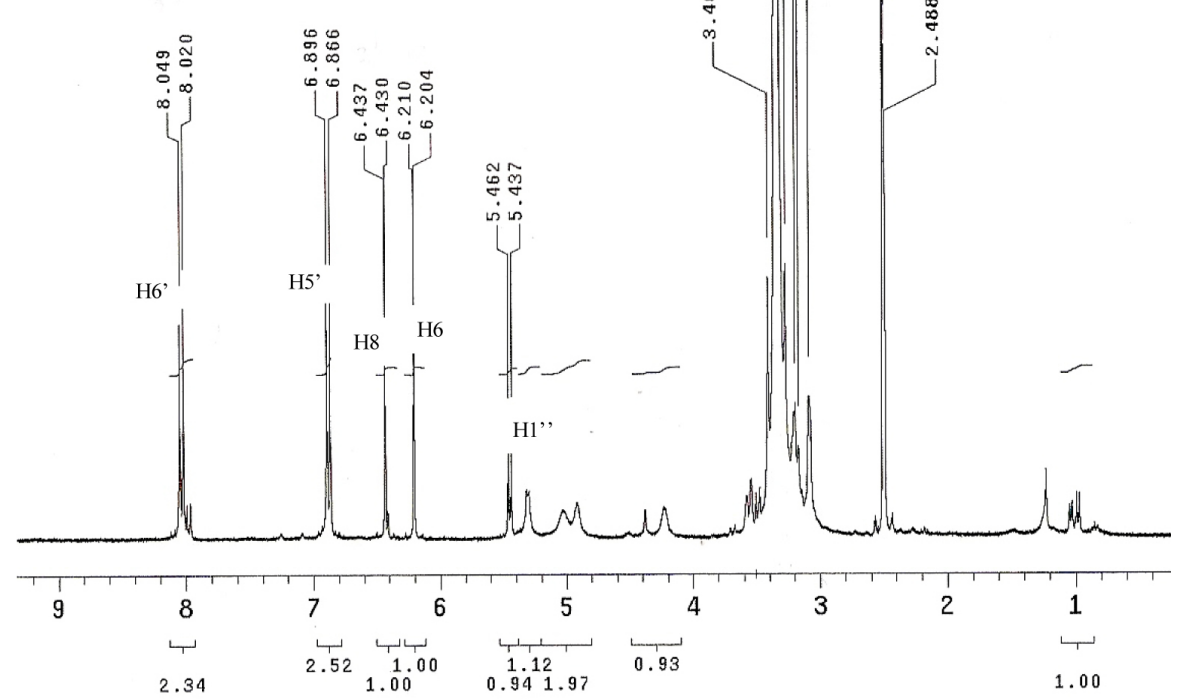

Figura 10S. Espectro de RMN de ${ }^{l} \mathrm{H}$ do kaempferol-3-O- $\beta$-D-glucopiranosideo (4)

Ácido gálico (5): RMN ${ }^{1} \mathrm{H}$ [500 MHz, DMSO-d ${ }_{6}, \delta$ (ppm), J (Hz)]: 6,92 (s, H-2 e H-6). RMN ${ }^{13} \mathrm{C}$ [125 MHz, DMSO-d $\left.{ }_{6}, \delta(\mathrm{ppm})\right]: 120,7$

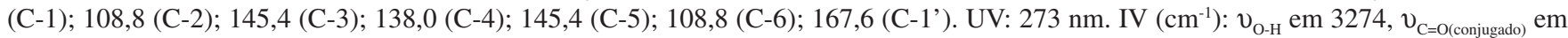
$1602, v_{\mathrm{C}=\mathrm{C}(\text { aromático })}$ em 1535 .

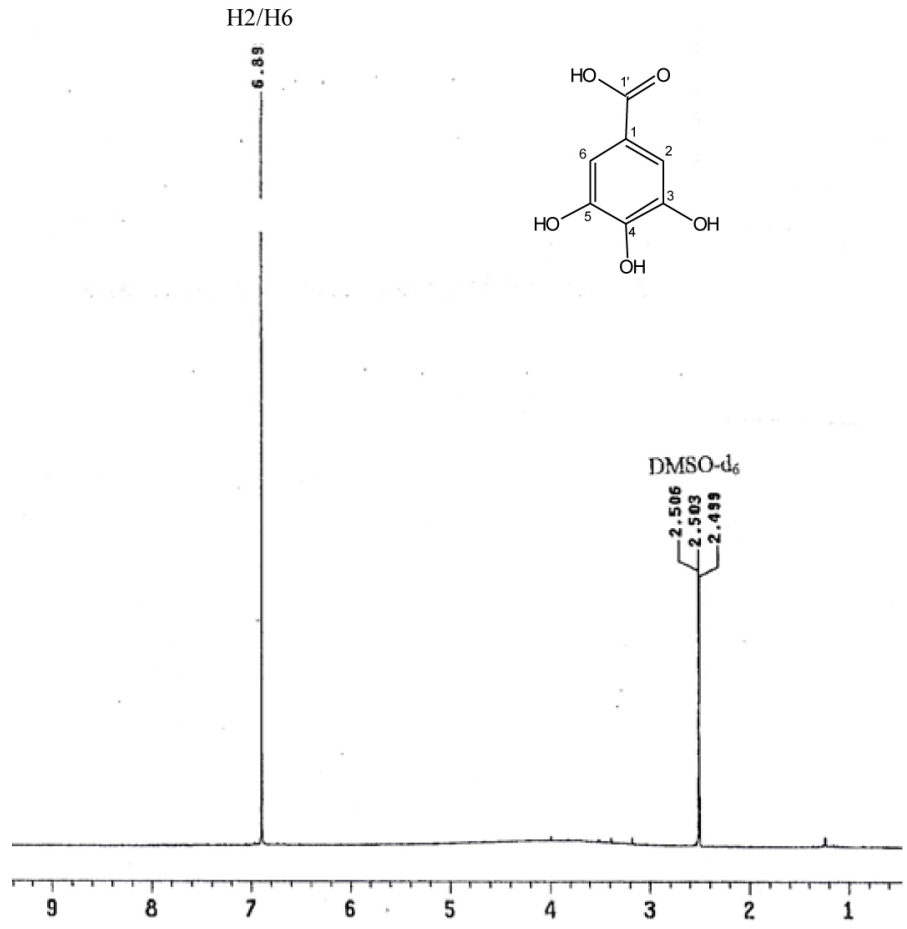

Figura 11S. Espectro de RMN de ${ }^{1} H$ do ácido gálico (5) 
Galato de metila (6): RMN ${ }^{1} \mathrm{H}\left[500 \mathrm{MHz}, \mathrm{DMSO}-\mathrm{d}_{6}, \delta(\mathrm{ppm}), \mathrm{J}(\mathrm{Hz})\right]:$ 6,93 (s, H-2 e H-6); 3,74 (s, $\mathrm{CH}_{3}$ ). RMN ${ }^{13} \mathrm{C}\left[125 \mathrm{MHz}, \mathrm{DMSO}-\mathrm{d}_{6}\right.$, $\delta(\mathrm{ppm})]: 119,7$ (C-1); 108,8 (C-2); 145,5 (C-3); 138,0 (C-4); 145,5 (C-5); 108,8 (C-6); 165,6 (C-1'); $52,3\left(\mathrm{OCH}_{3}\right) . \mathrm{UV}^{\prime}: 274$ nm. IV (cm-1): $v_{\mathrm{O}-\mathrm{H}}$ em $3293, v_{\mathrm{C}=\mathrm{O}(\text { conjugado) }}$ em 1674, $\mathrm{v}_{\mathrm{C}=\mathrm{C}(\text { aromático) }}$ em 1540.<smiles>COC(=O)c1cc(O)c(O)c(O)c1</smiles>

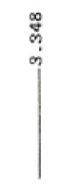

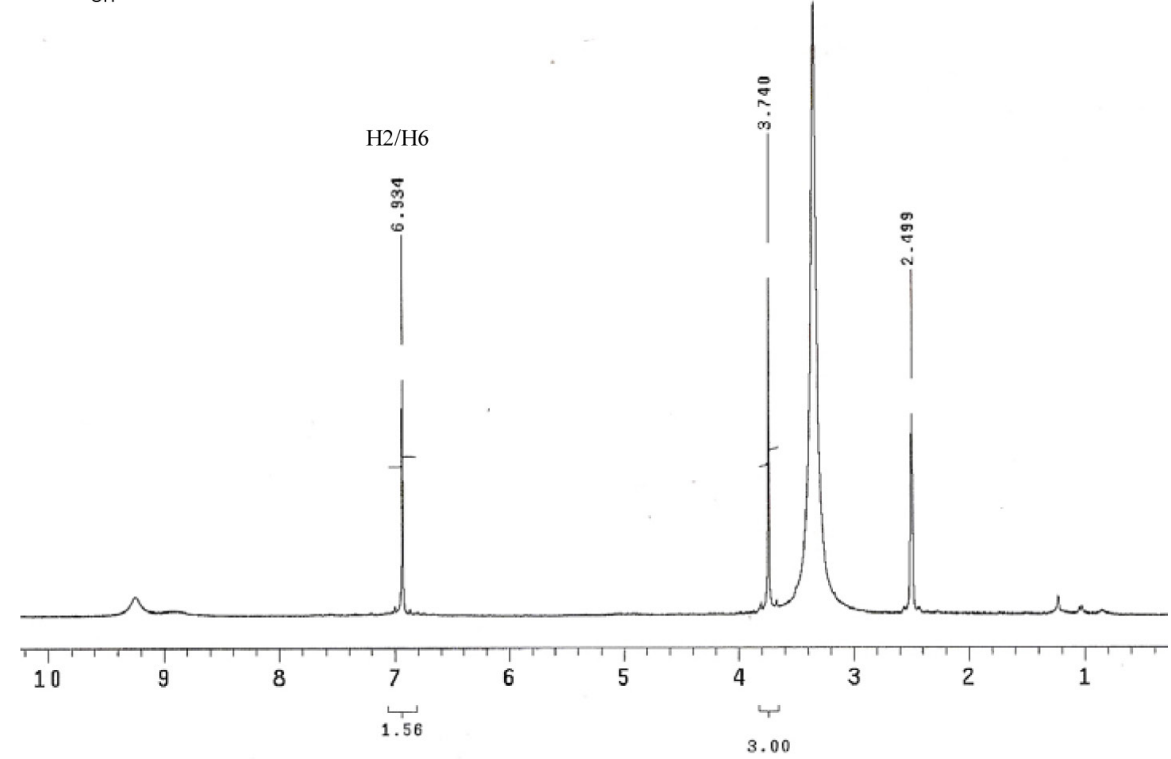

Figura 12S. Espectro de $R M N$ de ${ }^{1} \mathrm{H}$ do galato de metila $(\mathbf{6})$

Kaempferol (5): RMN ${ }^{1} \mathrm{H}\left[500 \mathrm{MHz}, \mathrm{DMSO}_{6}, \delta\right.$ (ppm), J (Hz)]: 6,20 (d, J=2,0 Hz, H-6); 6,44 (d, J=2,0 Hz, H-8); 8,05 (d, J=8,5 Hz, H-2'); 6, 94 (d, J=8,5 Hz, H-3'); 6,94 (d, J=8,5 Hz, H-5'); 8,05 (d, J=8,5 Hz, H-6'). RMN ${ }^{13} \mathrm{C}$ [125 MHz, DMSO-d,$\delta$ (ppm)]: 147,0 (C-2); 99,0 (C-6); 164,2 (C-7); 94,0 (C-8); 156,2 (C-9); 103,0 (C-10); 122,0 (C-1'); 130,0 (C-2'); 115,0 (C-3'); 159,0 (C-4'); 115,0 (C-5'); 130,0

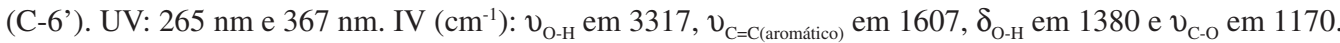

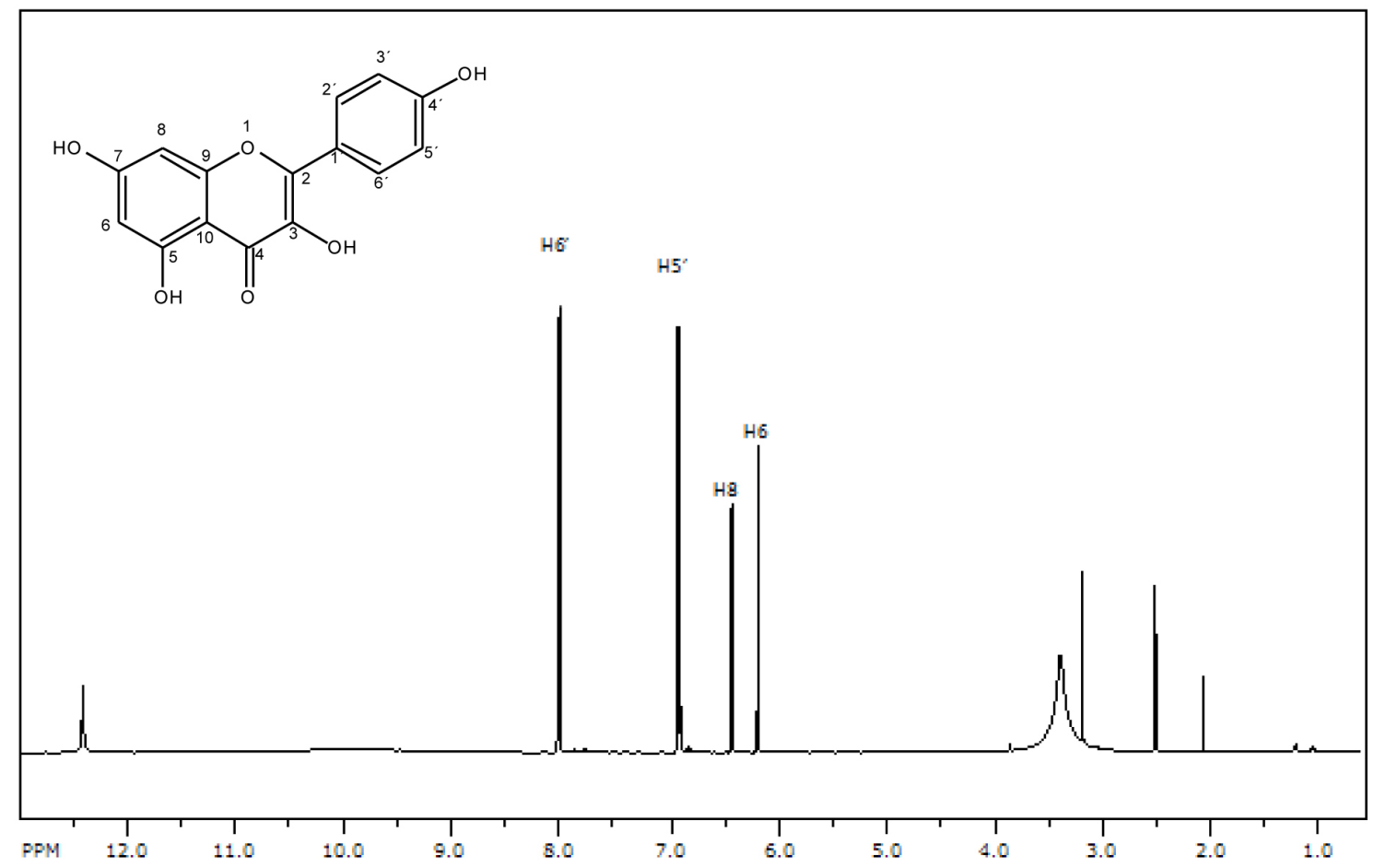

Figura 13S. Espectro de RMN de ${ }^{1} H$ do kaempferol (7) 
Ácido protocatecuico (8): RMN ${ }^{1} \mathrm{H}\left[500 \mathrm{MHz}, \mathrm{DMSO}-\mathrm{d}_{6}, \delta(\mathrm{ppm}), \mathrm{J}(\mathrm{Hz})\right]:$ 7,32 (d, J=1,5 Hz, H-2); 6,76 (d, J=8,5 Hz, H-5); 7,27 (dd, $\mathrm{J}=8,5$ e J=1,5 Hz, H-6). RMN ${ }^{13} \mathrm{C}$ [125 MHz, DMSO-d 6 , $\delta$ (ppm)]: 117,1 (C-2); 145,0 (C-3); 150,0 (C-4); 114,4 (C-5); 122,4 (C-6); 167,0 (C-1'). UV: $260 \mathrm{~nm}$ e $294 \mathrm{~nm}$. IV (cm-1): $v_{\mathrm{C}-\mathrm{H}(\text { aromático) }}$ em 3182, $v_{\mathrm{C}=\mathrm{O}(\text { conjugado) }}$ em 1673, $v_{\mathrm{C}=\mathrm{C}(\text { aromático) }}$ em 1597.

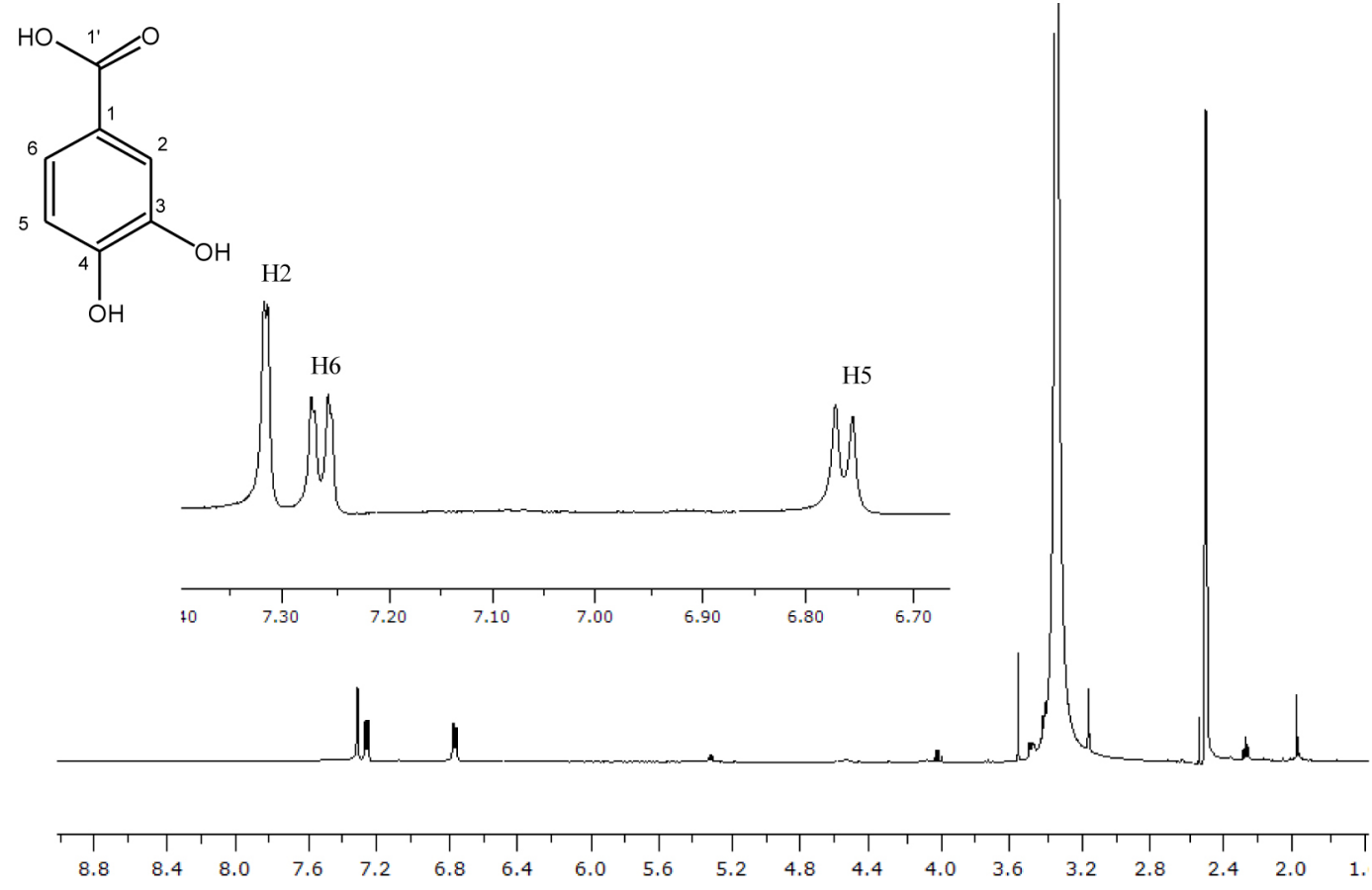

Figura 14S. Espectro de $R M N$ de ${ }^{1} \mathrm{H}$ do ácido protocatecuico $(\mathbf{8})$ 